\title{
XMM-Newton spectroscopy of an X-ray selected sample of RL AGNs $\star, \star \star$
}

\author{
E. Galbiati ${ }^{1,2}$, A. Caccianiga ${ }^{1}$, T. Maccacaro ${ }^{1}$, V. Braito $^{1}$, R. Della Ceca ${ }^{1}$, \\ P. Severgnini ${ }^{1}$, H. Brunner ${ }^{3}$, I. Lehmann ${ }^{3}$, and M. J. Page ${ }^{4}$ \\ e-mail: [galbiati;caccia; tommaso; braito;rdc;paola]@brera.mi.astro.it \\ 3 Max-Planck-Institut für extraterrestrische Physik, Giessenbachstrasse, 85748 Garching, Germany \\ 4 Mullard Space Science Laboratory, University College London, Holmbury St. Mary, Dorking, Surrey, RH5 6NT, UK
}

1 INAF - Osservatorio Astronomico di Brera, via Brera 28 - 20121 Milano, Italy

2 Dipartimento di Fisica, Università di Milano, Via Celoria 16, 20133 Milan, Italy e-mail: hbrunner@mpe.mpg.de; ile@mpe.mpg.de e-mail: mjp@mssl.ucl.ac.uk

\begin{abstract}
This paper presents the X-ray spectroscopy of an X-ray selected sample of 25 radio-loud (RL) AGNs extracted from the XMM-Newton Bright Serendipitous Survey (XBSS). The main goal of the work is to assess and study the origin of the X-ray spectral differences usually observed between radio-loud and radio-quiet (RQ) AGNs. To this end, a comparison sample of 53 RQ AGNs has been also extracted from the same XBSS sample and studied together with the sample of RL AGNs. Since there are many claims in the literature that RL AGNs have, on average, a flatter spectral index when compared to the RQ AGNs, we have focused the analysis on the distribution of the X-ray spectral indices of the power-law component that models the large majority of the spectra in both samples. We find that the mean X-ray energy spectral index is very similar in the 2 samples and close to $\alpha_{\mathrm{X}} \sim 1$. However, the intrinsic distribution of the spectral indices is significantly broader in the sample of RL AGNs. In order to investigate the origin of this difference, we have divided the RL AGNs into blazars (i.e. BL Lac objects and FSRQs) and "non-blazars" (i.e. radiogalaxies and SSRQs), on the basis of the available optical and radio information. Although the number of sources is small, we find strong evidence that the broad distribution observed in the RL AGN sample is mainly due to the presence of the blazars. Furthermore, within the blazar class we have found a link between the X-ray spectral index and the value of the radio-to-X-ray spectral index $\left(\alpha_{\mathrm{RX}}\right)$ suggesting that the observed X-ray emission is directly connected to the emission of the relativistic jet. This trend is not observed among the "non-blazars" RL AGNs. This favours the hypothesis that, in these latter sources, the X-ray emission is not significantly influenced by the jet emission and it has probably an origin similar to the RQ AGNs. Overall, the results presented here indicate that the observed distribution of the X-ray spectral indices in a given sample of RL AGNs is strongly dependent on the amount of relativistic beaming present in the selected sources, i.e. on the relative fraction of blazars and "non-blazars".
\end{abstract}

Key words. galaxies: nuclei - X-rays: galaxies - galaxies: BL Lacertae objects: general

\section{Introduction}

The origin of the differences between radio-loud (RL) and radio-quiet (RQ) AGNs is one of the fundamental questions in the AGN phenomenology. The broad and possibly bimodal distribution of the radio-to-optical flux ratios observed within the class of AGNs (e.g. Kellermann et al. 1989;

* Based on observations collected at the Telescopio Nazionale Galileo (TNG) and at the European Southern Observatory (ESO), La Silla, Chile and on observations obtained with XMM-Newton, an ESA science mission with instruments and contributions directly funded by ESA Member States and the USA (NASA).

$\star \star$ Appendix $\mathrm{A}$ is only available in electronic form at http://www. edpsciences.org
Della Ceca et al. 1994; Ivezic et al. 2002; but see also White et al. 2000; Cirasuolo et al. 2003, for evidences against the bimodality) is not yet fully understood and it may reflect important differences between the physical processes at work in the inner regions of the 2 classes of objects. It has been proposed that the "radio-loudness" of the AGNs could be directly connected to the mass of the central black-hole (Franceschini et al. 1998; Laor 2000) or to its spin (e.g. Meier 1999). Alternatively, a different accretion rate (e.g. Ho 2002) or a different structure of the accretion disk (e.g. see Ballantyne et al. 2002 and references therein) may be at the origin of the observed phenomenology.

In this context, X-ray observations can play a key role since they bring information on the innermost region of 
the AGN structure. In particular, a direct comparison between the X-ray properties of RL and RQ AGNs can shed light on the origin of the "radio-loudness".

If the main properties of the X-ray spectrum of RQ AGNs are quite well established, RL AGNs are, in general, less studied. Early observations carried out with Einstein have suggested that RL AGNs have, on average, a flatter spectrum than RQ AGNs (e.g. Wilkes \& Elvis 1987; Shastri et al. 1993). This difference is more evident if only RL AGNs with a flat radio spectrum (i.e. the Flat Spectrum Radio Quasars, FSRQs) are considered, while it becomes marginal when only the AGNs with a steep radio spectrum (i.e. the Steep Spectrum Radio Quasars, SSRQs) are compared to the RQ AGNs (Canizares \& White 1989). This trend has been subsequently confirmed with ASCA and BeppoSAX data: on the one hand, the X-ray spectrum of a sample of RL AGNs (mostly composed by FSRQs) studied by Reeves et al. (1997) and Reeves \& Turner (2000) turned out to be significantly flatter, on average, when compared to RQ AGNs; on the other hand, this difference in the $\mathrm{X}$-ray spectral index is not clearly present in the samples studied by Sambruna et al. (1999) and Hasenkopf et al. (2002), mostly composed by radiogalaxies or lobe-dominated quasars.

Apart from the spectral index, other differences in the X-ray spectra of RL and RQ AGNs include a weaker "reflection" hump and Fe I emission line in RL AGNs (e.g. Hasenkopf et al. 2002; Eracleous et al. 2000; Sambruna et al. 1999; Wozniak et al. 1998). These results have been interpreted as related to a different structure of the accretion disk in RL AGNs (e.g. Ballantyne et al. 2002). Moreover, Sambruna et al. (1999) have pointed out that among RL AGNs, the presence of a thermal component (from hot plasma related to the host galaxy and/or to a cluster of galaxies) is very common.

Altogether, the data collected so far seem to indicate that there are significant differences in the X-ray spectrum of RL and RQ AGNs although the details of these differences depend on the radio spectral index of the source. In particular, the presence of a significant contribution to the X-ray emission coming from the relativistic jets can play an important role in some RL AGNs (like the FSRQs).

Chandra and XMM-Newton can give a fundamental contribution to this topic: different components of the X-ray emission in RL AGNs can be resolved spatially, with Chandra, (e.g. Gambill et al. 2003 or Schwartz 2004 for a review) or spectrally, with XMM-Newton (e.g. Brocksopp et al. 2004; Ferrero \& Brinkmann 2003; Page et al. 2004). In this paper, we present a systematic X-ray spectral analysis of an X-ray selected sample of 25 RL AGNs extracted from the XMM-Newton Bright Serendipitous Survey (XBSS, Della Ceca et al. 2004). In this sample we have distinguished between the AGNs with flat and steep radio spectrum in order to establish the importance of the jets in the X-ray emission of RL objects. In order to assess how different the selected RL AGNs are with respect to the RQ AGNs, we have also selected from the same XBSS a "comparison" sample of 53 objects, mostly composed by RQ AGNs. Since the two samples have a common origin, the only difference being the radio emission, we expect that any observed difference could be connected to the different mechanisms at work in the 2 classes of AGNs.
Throughout this paper $H_{0}=65 \mathrm{~km} \mathrm{~s}^{-1} \mathrm{Mpc}^{-1}, \Omega_{\Lambda}=0.7$ and $\Omega_{\mathrm{m}}=0.3$ are assumed.

\section{The XBSS sample of RL AGNs}

The XBSS (Della Ceca et al. 2004) consists of two flux-limited samples selected in the $0.5-4.5 \mathrm{keV}$ and $4.5-7.5 \mathrm{keV}$ energy bands respectively from the analysis of 237 XMM-Newton archive images. The flux limit, in both energy bands, is relatively bright $\left(\sim 7 \times 10^{-14} \mathrm{erg} \mathrm{cm}^{-2} \mathrm{~s}^{-1}\right)$ so as to allow an accurate analysis of all the selected sources, both from the X-ray and the optical point of view. The sample selected in the $0.5-4.5 \mathrm{keV}$ energy band, in particular, contains 389 sources and it is well suited to extract and to study a sub-sample of RL AGNs.

Given the relatively bright X-ray flux limit, about $90 \%$ of the optical counterparts of the X-ray sources are brighter than mag $=21$. At these magnitudes, the majority of RL AGNs are expected to have a radio flux at the $\sim$ mJy level at $\sim \mathrm{GHz}$ frequencies given the typical radio-to-optical flux ratios of the RL AGNs (see next section). Therefore, a positional crosscorrelation of the XBSS sample with one of the existing wide-angle radio surveys (NVSS, Condon et al. 1998, or FIRST, Becker et al. 1995) allows the selection of most of the RL AGNs present in the sample. Although the NVSS survey is less deep ( $\sim 2.5 \mathrm{mJy})$ than the FIRST survey $(\sim 1 \mathrm{mJy})$, it covers a larger area of sky $\left(\sim 34000 \mathrm{Deg}^{2}\right)$ offering the availabilty of radio data (either a detection or an upper limit) for $\sim 85 \%$ of the XBSS sources.

We have thus cross-correlated the XBSS sample with the NVSS. In total, there are 332 sources (out of 389) in the XBSS that fall in the area of sky covered by the NVSS. Given the good radio (see Condon et al. 1998) and X-ray (see Della Ceca et al. 2004) positions we have established that a positional tolerance of $10^{\prime \prime}$ is large enough to find all the radio counterparts of the X-ray sources except for the objects that are resolved, in the radio, in two or more components (e.g. the radio galaxies). The positional cross-correlation with a tolerance of $10^{\prime \prime}$ has produced $28 \mathrm{X}$-ray/radio matches. For completeness we have subsequently run an additional cross-correlation, like the one used to select the sources of the REX survey (Caccianiga et al. 1999), specifically sensitive to complex morphologies (e.g. double or triple radio sources). However, no sources of this type have been found. This is consistent with the results of the REX survey, where the sources with a complex radio morphology (in the NVSS) represent only $\sim 5 \%$ of the total radio/X-ray matches at these frequencies and flux limits (Caccianiga et al. 1999), which corresponds to $\sim 1$ source of this type expected in the XBSS sample.

In order to estimate the reliability of the radio/X-ray matches, we have computed the expected number of random matches within $10^{\prime \prime}$ using the source density given by Condon et al. (1998). We expect $\sim 0.4$ spurious matches in the sample which implies that one source, at most, could be a chance radio/X-ray match. As a further confirmation of this result we have also performed the same cross-correlation after having positionally shifted the X-ray catalogue along the declination so that only chance coincidences are expected. We have repeated 11 times the cross-correlation between the two catalogues 
with positional offsets ranging from 10 to 110 arcmin. All these 11 cross-correlations have produced 0 matches thus confirming the negligible number of expected spurious matches.

In conclusion, we have found 28 sources, out of the 332 XBSS objects included in the NVSS sky coverage, that have a flux density at $1.4 \mathrm{GHz}$ larger than $2.5 \mathrm{mJy}$. The list of these $28 \mathrm{X}$-ray/radio matches is reported in Table 1.

\subsection{The radio-loudness parameter}

The simple detection of a source in the NVSS catalogue does not necessarily imply that the object is radio-loud, given the range of magnitudes of the selected sources. At the same time, the NVSS flux limit is not deep enough to guarantee the detection of all the radio-loud AGNs present in the XBSS sample. We have thus analysed the radio-loudness parameter $(R)$ of the 28 sources and the distribution of the upper limits on $R$ of the 304 XBSS sources not detected in the NVSS in order to pinpoint all RL AGNs actually selected, on the one hand, and to estimate the fraction of RL AGNs that are lost in the crosscorrelation because of the NVSS flux limit, on the other hand.

The parameter $R$ is defined as (Kellermann et al. 1989):

$R=\frac{S_{5 \mathrm{GHz}}}{S_{4400 \AA}}$

where $S_{5 \mathrm{GHz}}$ is the radio monochromatic flux at $5 \mathrm{GHz}$ and $S_{4400 \AA}$ is the optical monochromatic flux at $4400 \AA$. The radio flux at $5 \mathrm{GHz}$ has been derived from the NVSS flux at $1.4 \mathrm{GHz}$ and $\mathrm{K}$-corrected by assuming a mean radio spectral index of 0.7 ( $S \propto v^{-\alpha}$, Ciliegi et al. 2003). The optical flux is derived from the optical magnitude taken from APM (Automatic Plate Measuring machine) catalogue ${ }^{1}$ or from the literature and $\mathrm{K}$-corrected assuming an optical spectral index of 0.5 (Ciliegi et al. 2003). A magnitude has been obtained for 25 out of 28 sources. The remaining 3 objects are blankfields on the DSS material. For these objects the $R$ parameter is computed assuming a magnitude equal to the DSS limit $(B \sim 22)$. In these cases, the actual $R$ parameter is expected to be larger than the computed value. However, based on what we have found already in the total XBSS sample, we expect that the optical counterpart in these 3 objects is close to the DSS limit so that the true value of $R$ should be close to the computed lower limit. In any case, these 3 sources have a lower limit on $R$ well above the radio-loud limit ( $R=10$, see below) so that their classification as RL objects is firm and does not depend on the exact value of the magnitude.

We finally note that we have used the total magnitude for the computation of the $R$ parameter without attempting to separate the contribution due to the host galaxy. The importance of the correct estimate of the optical nuclear emission in the computation of the radio-loudness has been often stressed in the literature (e.g. Ho \& Peng 2001). However, this problem is really critical for samples containing mostly low-luminosity AGN, for which the emission from the host galaxy is always relevant, if not dominant. This is not the case with the sample discussed

\footnotetext{
${ }^{1}$ http://www.ast.cam.ac.uk/ apmcat/
}

here, which should be only marginally affected by this problem. In any case, the computed $R$ parameter for the few objects in which the host galaxy is important should be more correctly regarded as a lower limit.

In Fig. 1 we show the distribution of the $R$ parameter for the $28 \mathrm{X}$-ray/radio matches and the distribution of the upper limits on $R$ for the $304 \mathrm{X}$-ray sources (falling in the area of sky covered by the NVSS) not detected in the radio band. Out of 28 radio/X-ray matches, 25 are radio-loud, using a defining limit of $R=10$. We stress here that sources with such a large radio-to-optical flux ratio typically contain relativistic jets, i.e. an AGN. Indeed, based on the available optical, radio or X-ray information, the presence of an AGN is confirmed, or strongly suggested, in all these 25 objects (see next section). For this reason we will use here the term "RL AGN" for all the objects with $R>10$.

We have then used the non-parametric method described by Avni et al. (1980) to estimate the real distribution of $R$ for the sample XBSS. This method provides an analytic solution for the best estimate of a distribution function of one (binned) independent variable (the $R$ parameter) taking into account the upper limits, under the assumption that these are distributed like the detections. Based on the distribution of $R$, derived with the Avni et al. (1980) method, we have estimated that about 10 RL AGNs present in the XBSS sample have not been detected in the NVSS because the flux limit is not deep enough $^{2}$. Therefore, the expected number of RL AGNs among the 332 XBSS sources covered by the NVSS data is about 35 (25 detected and $\sim 10$ not-detected) which corresponds to a fraction of $10.5 \%$ of the X-ray sources. Since the fraction of AGNs in the XBSS sample is about $81 \%$ (Della Ceca et al. 2004), the fraction of RL AGNs among the total population of X-ray selected AGNs at the survey flux limit is $\sim 13 \%$. This fraction is consistent with what has been found in previous X-ray surveys, like the EMSS survey (Della Ceca et al. 1994), at similar flux limits.

In the rest of the paper we will consider only the sample of RL AGNs, i.e. the one composed by 25 objects with $R>10$.

\section{Optical classification}

For the majority (20) of the 25 sources in the sample we have collected a spectral classification, based either on our own optical spectroscopy or from the literature. According to the classification criteria described in Della Ceca et al. (2004) we have broadly divided the sources into AGN1 (Broad Line AGNs) with broad emission lines ( $\left.F W H M>1000 \mathrm{~km} \mathrm{~s}^{-1}\right)$ in the optical spectrum, AGN2 (Narrow Line AGNs) showing only narrow emission lines $\left(F W H M<1000 \mathrm{~km} \mathrm{~s}^{-1}\right)$ and objects without strong $(E W<5 \AA)$ emission lines in the spectrum.

In particular, the AGN1 class includes QSOs, Broad Line radio galaxies, Sy1 and Narrow Line Seyfert 1 (NLSy1) while

\footnotetext{
${ }^{2}$ Indeed, among the XBSS sources that have not been detected in the NVSS we have found one object optically classified as BL Lac, which is expected to belong to the radio-loud population. Deeper radio observations, reaching a limit of $\sim 0.2 \mathrm{mJy}$, should be able to detect all the radio-loud sources in the sample.
} 
Table 1. The 28 sources resulting from the X-ray/radio correlation.

\begin{tabular}{|c|c|c|c|c|c|c|c|c|c|c|}
\hline $\begin{array}{l}\text { Name } \\
\text { (1) }\end{array}$ & $\begin{array}{l}\mathrm{CR} \\
\text { (2) }\end{array}$ & $\begin{array}{r}S_{1.4}^{\mathrm{int}} \\
(3)\end{array}$ & $\begin{array}{r}S_{1.4}^{\mathrm{pk}} \\
(4)\end{array}$ & $\begin{array}{r}\text { mag } \\
(5)\end{array}$ & $\begin{array}{l}\text { Class } \\
(6)\end{array}$ & $\begin{array}{l}\text { Redshift } \\
\text { (7) }\end{array}$ & $\begin{array}{l}\alpha_{R} \\
(8)\end{array}$ & $\begin{array}{l}\text { Radio class } \\
\text { (9) }\end{array}$ & $\begin{array}{r}\log (R) \\
(10)\end{array}$ & $\begin{array}{r}\mathrm{cd} \\
\text { (11) }\end{array}$ \\
\hline XBSJ000100.2-250501 & 0.014 & 130.0 & 109.9 & 21.8 & $\mathrm{AGN}^{1}{ }^{1}$ & 0.85 & $>0.89^{a}$ & $\mathrm{nb}\left(\alpha_{\mathrm{R}}\right)$ & 3.85 & - \\
\hline XBSJ003255.9+394619 & 0.011 & 38.8 & 33.6 & 18.7 & AGN1 & 1.14 & $0.58^{b}$ & $\mathrm{~b}\left(\alpha_{\mathrm{R}}\right)$ & 2.12 & - \\
\hline XBSJ012505.4+014624 & 0.019 & 186.6 & 186.6 & 19.6 & AGN1 & 1.56 & $0.51^{c}$ & $\mathrm{~b}\left(\alpha_{\mathrm{R}} ; \mathrm{L} 1\right)$ & 3.25 & - \\
\hline XBSJ012654.3+191246 & 0.010 & 1408.1 & 947.5 & $15.2^{l}$ & $\mathrm{G}$ & 0.04 & $0.53^{c}$ & $\mathrm{nb}(\mathrm{L} 2)$ & 2.10 & 29.80 \\
\hline XBSJ013240.1-133307 & 0.014 & 4.6 & 4.6 & 21.4 & AGN2 & 0.56 & $>-2.06^{a}$ & $?$ & 2.31 & - \\
\hline XBSJ013811.7-175416 & 0.031 & 15.6 & 15.6 & 20.8 & BL? & 0.50 & $>-1.14^{a}$ & $\mathrm{~b}(\mathrm{O})$ & 2.60 & - \\
\hline XBSJ021640.7-044404 & 0.026 & 88.3 & 88.3 & 16.9 & AGN1 & 0.87 & $0.95^{d}$ & $\mathrm{nb}\left(\alpha_{\mathrm{R}}\right)$ & 1.79 & - \\
\hline XBSJ033226.9-274107 & 0.014 & 22.5 & 19.6 & 19.2 & AGN1 & 0.73 & $>-0.53^{a}$ & $?$ & 2.05 & - \\
\hline XBSJ052108.5-251913 & 0.035 & 25.1 & 19.6 & 17.7 & AGN1 & 1.20 & $>-0.38^{a}$ & $?$ & 1.48 & - \\
\hline XBSJ061342.7+710725 & 0.064 & 25.2 & 25.2 & $19.6^{l}$ & $\mathrm{BL}^{2}$ & 0.27 & $0.01^{e}$ & $\mathrm{~b}\left(\mathrm{~L} 3 ; \alpha_{\mathrm{R}}\right)$ & 2.31 & - \\
\hline XBSJ084026.2+650638 & 0.012 & 67.6 & 49.2 & 21.5 & AGN1 & 1.14 & $0.92^{b}$ & $\mathrm{nb}\left(\alpha_{\mathrm{R}}\right)$ & 3.42 & - \\
\hline XBSJ095218.9-013643* & 0.090 & 62.2 & 62.2 & $14.4^{l}$ & $\mathrm{AGN} 1^{3}$ & 0.02 & $>0.35^{a}$ & RQ & 0.59 & 49.28 \\
\hline XBSJ095341.1+014204* & 0.163 & 9.6 & 9.6 & $17.1^{l}$ & $\mathrm{CL}$ & 0.10 & $>-0.95^{a}$ & RQ & 0.85 & 10.31 \\
\hline XBSJ101511.8+520708 & 0.017 & 77.5 & 77.5 & 21.6 & AGN1 & 0.89 & $0.90^{b}$ & $\mathrm{nb}\left(\alpha_{\mathrm{R}}\right)$ & 3.63 & 8.72 \\
\hline XBSJ101706.5+520245 & 0.018 & 39.7 & 39.7 & 19.9 & $\mathrm{BL}$ & 0.38 & $0.51^{f}$ & $\mathrm{~b}\left(\mathrm{O} ; \alpha_{\mathrm{R}}\right)$ & 2.62 & 4.01 \\
\hline XBSJ102016.1+082143 & 0.011 & 3.7 & 3.7 & $>22.0$ & - & - & $>-1.5^{a}$ & $?$ & $>2.41$ & 0.89 \\
\hline XBSJ111654.8+180304* & 0.013 & 6.9 & 6.9 & $10.8^{l}$ & $\mathrm{AGN}^{4}$ & 0.003 & $>-0.84^{a}$ & RQ & -1.80 & 5.94 \\
\hline XBSJ111928.5+130250 & 0.018 & 40.0 & 14.9 & 18.4 & AGN1 & 2.39 & $>0.47^{a}$ & $\mathrm{nb}(\mathrm{M})$ & 1.80 & 0.44 \\
\hline XBSJ122658.1+333246 & 0.072 & 4.3 & 4.3 & $>22.0$ & $\mathrm{CL}^{5}$ & 0.89 & $0.94^{f}$ & $\mathrm{nb}\left(\alpha_{\mathrm{R}}\right)$ & $>2.53$ & 360.00 \\
\hline XBSJ123538.6+621644 & 0.021 & 3.7 & 3.7 & 18.9 & AGN1 & 0.71 & $2.5^{h}$ & $\mathrm{nb}\left(\alpha_{\mathrm{R}}\right)$ & 1.22 & 14.62 \\
\hline XBSJ124903.6-061049 & 0.025 & 12.6 & 12.6 & 19.2 & AGN1 & 0.61 & $>-0.91^{a}$ & $\mathrm{nb}(\mathrm{M})$ & 1.88 & 0.59 \\
\hline XBSJ132105.5+341459 & 0.027 & 473.6 & 446.5 & 20.4 & - & - & $0.82^{g}$ & $\mathrm{nb}\left(\alpha_{\mathrm{R}} ; \mathrm{M}\right)$ & 3.86 & 0.44 \\
\hline XBSJ133026.6+241520 & 0.021 & 46.4 & 41.9 & 20.5 & - & - & $0.29^{e}$ & $\mathrm{~b}\left(\alpha_{\mathrm{R}}\right)$ & 2.87 & 0.79 \\
\hline XBSJ133232.6+111220 & 0.011 & 26.0 & 26.0 & $>22.0$ & & - & $>0.06^{a}$ & $?$ & $>3.26$ & 8.31 \\
\hline XBSJ153452.3+013104 & 0.094 & 1320.4 & 1320.4 & 19.8 & $\mathrm{AGN}^{6}{ }^{6}$ & 1.43 & $0.02^{e}$ & $\mathrm{~b}\left(\alpha_{\mathrm{R}} ; \mathrm{L} 4\right)$ & 4.15 & 24.25 \\
\hline XBSJ164237.9+030014 & 0.012 & 45.5 & 45.5 & 18.6 & - & - & $0.26^{e}$ & $\mathrm{~b}\left(\alpha_{\mathrm{R}}\right)$ & 2.14 & - \\
\hline XBSJ221951.6+120123 & 0.013 & 5.8 & 5.8 & $21.3^{j}$ & AGN2 & 0.53 & $>-1.01^{a}$ & $?$ & 2.36 & - \\
\hline XBSJ235036.9+362204 & 0.024 & 316.5 & 273.3 & 19.3 & $\mathrm{BL}^{7}$ & 0.32 & $0.40^{e}$ & $\mathrm{~b}\left(\mathrm{~L} 1, \alpha_{\mathrm{R}}\right)$ & 3.22 & - \\
\hline
\end{tabular}

Column 1: source name (the asterisk indicates that the source is $\mathrm{RQ}, \log (R)<1$, and not considered anymore during the analysis);

Col. 2: $0.5-4.5 \mathrm{keV}$ count-rate (counts $\mathrm{s}^{-1}$ ) (see Della Ceca et al. 2004 for details);

Col. 3: integrated flux (NVSS) @ $1.4 \mathrm{GHz}[\mathrm{mJy}]$;

Col. 4: peak flux density (NVSS)@ $1.4 \mathrm{GHz}[\mathrm{mJy} / \mathrm{beam}]$;

Col. 5: blue magnitude from APM unless specified: ${ }^{l}$ blue magnitude from the literature; ${ }^{j}$ from APM red magnitude and assuming the average colour observed for the other sources in the sample $(\mathrm{O}-\mathrm{E}=1.3)$;

Col. 6: spectroscopic classification (AGN1 = type 1 AGN; AGN2 = type 2 AGN; G = galaxy; BL = BL Lac; CL = cluster of galaxies). Identification and $\mathrm{z}$ are from our own spectroscopy except for: ${ }^{1}$ Fiore et al. (2003); ${ }^{2}$ Morris et al. (1991); ${ }^{3}$ Nagao et al. (2001); ${ }^{4}$ Ho et al. (1997); ${ }^{5}$ Ebeling et al. (2001); ${ }^{6}$ Visvanathan \& Wills (1998); ${ }^{7}$ Perlman et al. (1998);

Col. 7: redshift;

Col. 8: radio spectral index or lower limit $\left(S \propto v^{-\alpha_{R}}\right) ;{ }^{a}$ calculated from $1.4 \mathrm{GHz}$ and an upper limit for the flux @ $5 \mathrm{GHz}$; ${ }^{b}$ is the mean of the values calculated between $1.4 \mathrm{GHz}$ and other 2 frequencies $\left(5 \mathrm{GHz}\right.$ and $325 \mathrm{MHz}$ ); ${ }^{c}$ is the mean of the values calculated between $1.4 \mathrm{GHz}$ and other 2 frequencies $\left(5 \mathrm{GHz}\right.$ and $365 \mathrm{MHz}$ ); ${ }^{d}$ calculated between $365 \mathrm{MHz}$ and $1.4 \mathrm{GHz} ;{ }^{e}$ calculated between $1.4 \mathrm{GHz}$ and $5 \mathrm{GHz} ;{ }^{f}$ calculated between $325 \mathrm{MHz}$ and $1.4 \mathrm{GHz} ;{ }^{g}$ is the mean of the values calculated between $1.4 \mathrm{GHz}$ and other $3 \mathrm{frequencies}(5 \mathrm{GHz}, 325 \mathrm{MHz}$ and $365 \mathrm{MHz}) ;{ }^{h}$ calculated between $1.4 \mathrm{GHz}$ and $8.5 \mathrm{GHz}$;

Col. 9: radio classification ( $\mathrm{b}=$ blazar, $\mathrm{nb}=$ non blazar, $\mathrm{RQ}=$ radio quiet). " $\alpha_{R}$ ": from the radio spectral index; " $\mathrm{M}$ ": from the radio morphology; "O": from the optical classification; "L": from the literature, i.e. 1 = Perlman et al. (1998), 2 = Owen \& Ledlow (1997), 3 = Morris et al. (1991), 4 = Visvanathan \& Wills (1998);

Col. 10: radio loudness parameter;

Col. 11: core dominance parameter. 


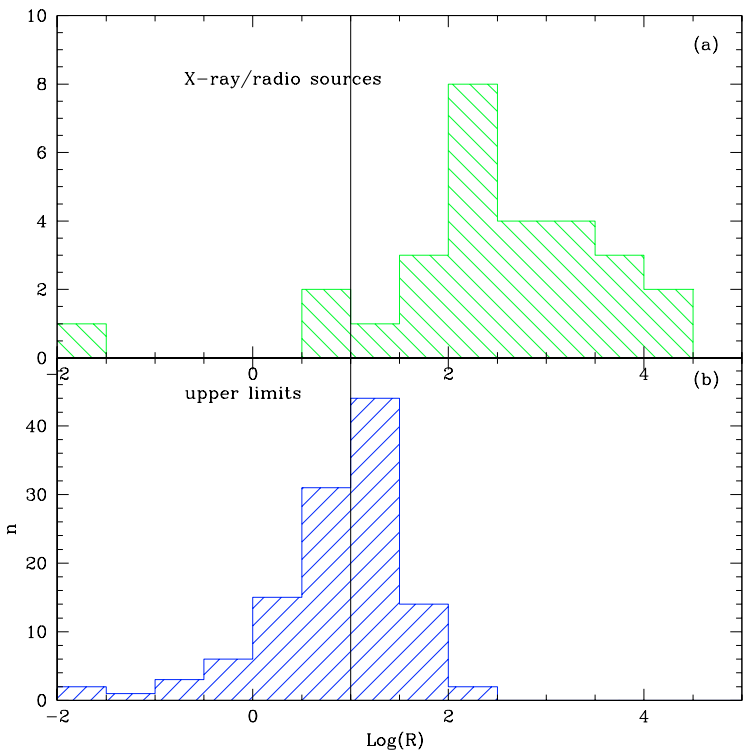

Fig. 1. Distribution of the radio-loudness parameter $(R)$ for the $28 \mathrm{X}$-ray/radio matches (panel a)) and the upper limits of $R$ for the XBSS sources not detected in the NVSS (panel b)).

the AGN2 class includes type2 QSO, Sy1.8, Sy1.9, Sy2 and Narrow Line radio galaxies. Finally, the class of objects with no emission lines includes BL Lac objects and "normal" galaxies.

For the classification of the objects with no emission lines in the optical spectrum, we have used the usual criteria based on the $4000 \AA$ break adopted in the major blazar surveys (e.g. the 200 mJy survey, Marchã et al. 1996; the RGB survey, Laurent-Muehleisen et al. 1998; the DXRBS survey, Perlman et al. 1998; Landt et al. 2001; the REX survey, Maccacaro et al. 1998, Caccianiga et al. 1999; the CLASS survey, Marchã et al. 2001; Caccianiga et al. 2001). In particular, we classify a source as BL Lac object if the $4000 \AA$ break is absent (i.e. a completely featureless spectrum) or below $40 \%$. Sources with a $4000 \AA$ break larger than $40 \%$ are classified as "normal" elliptical galaxies. A discussion on the physical meaning of these classification criteria is presented in Landt et al. (2002).

The optical spectra of the 2 newly discovered BL Lacs, taken at the Telescopio Nazionale Galileo (TNG) in September 2003 and December 2002, are presented in Fig. 2. The optical spectrum of the "normal" galaxy, taken at the Calar Alto $2.1 \mathrm{~m}$ telescope in October 2002, is reported in Fig. 3.

In conclusion, the optical spectrum, when available, reveals the presence of an AGN in 18 out of 20 objects (see Table 2). In the 2 objects, classified respectively as "normal galaxy" and "cluster of galaxy", the radio-power is large $\left(P_{1.4 \mathrm{GHz}}=\right.$ $6 \times 10^{24} \mathrm{~W} \mathrm{~Hz}^{-1}$ and $1.6 \times 10^{25} \mathrm{~W} \mathrm{~Hz}^{-1}$ respectively) thus confirming, also in these 2 objects, the presence of an AGN (i.e. a radiogalaxy isolated or in cluster, respectively). Similarly to the XBSS sources discussed in Severgnini et al. (2003), the optical signs of nuclear activity in these 2 radiogalaxies are probably diluted by the light of the host galaxy. Finally, in the 5 sources without an optical spectrum, the presence of an AGN is inferred by the X-ray spectra (see Sect. 5). We conclude that, as expected, all the sources with $R>10$ are hosting an AGN.

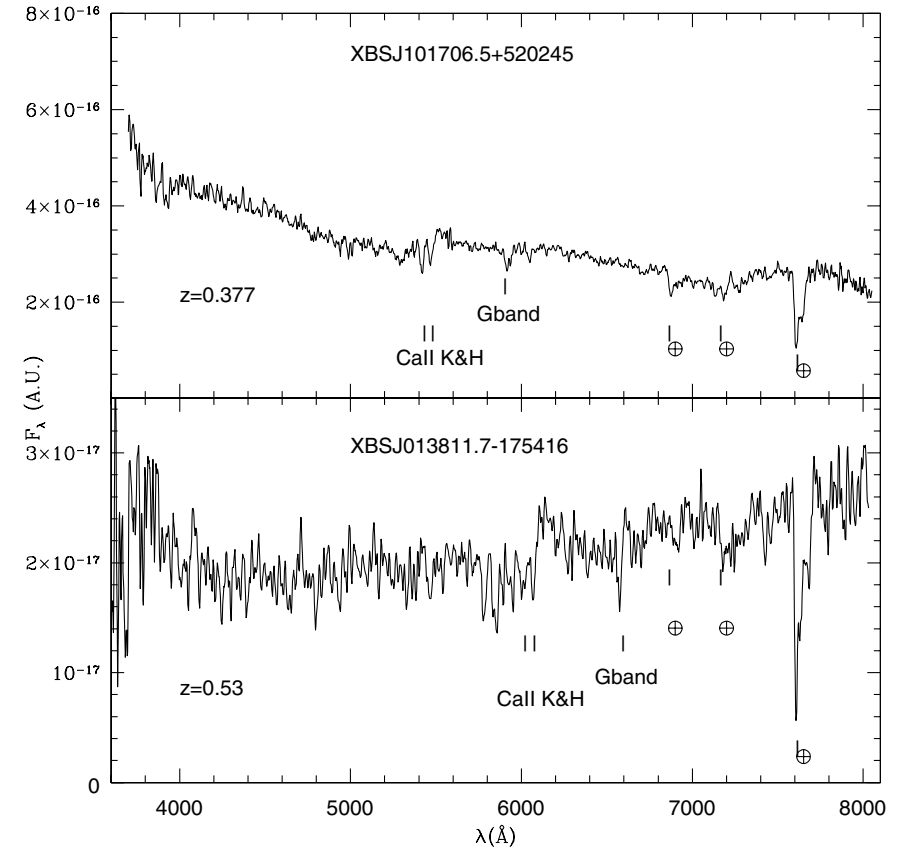

Fig. 2. Optical spectra of the 2 newly discovered BL Lacs.

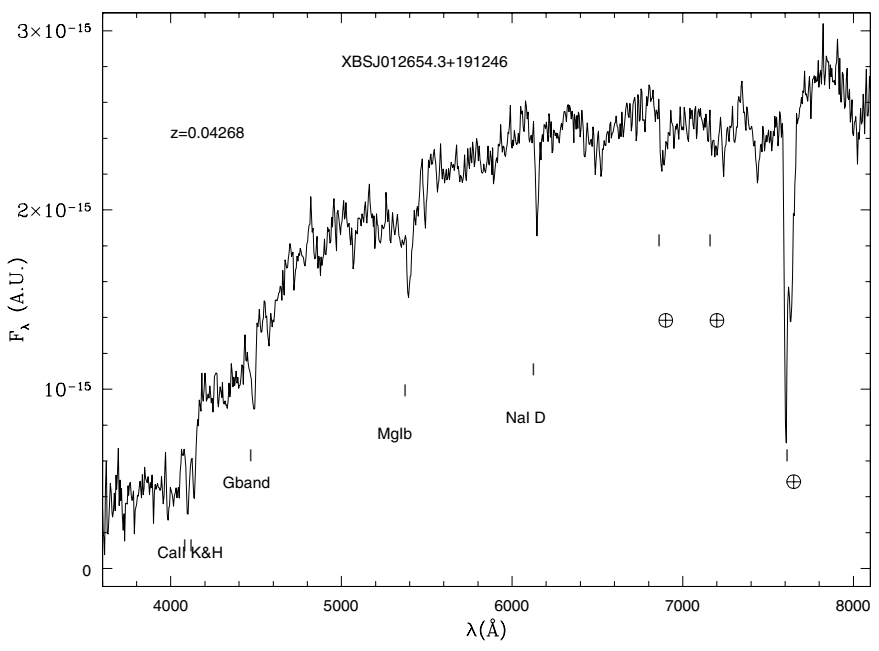

Fig. 3. Optical spectrum of the source classified as "normal" galaxy (a radiogalaxy).

\section{Radio classification}

In order to further characterize the sources we have collected all the radio data available from the literature. The main goal is to distinguish between core-dominated, flat spectrum objects (e.g. blazars) and lobe-dominated, steep spectrum sources (e.g. radio-galaxies and Steep Spectrum Radio Quasars, SSRQs). There are indications in the literature, in fact, that the emission observed in the X-ray band can be originated in different regions of the AGN depending on the radio classification of the object: in the case of blazars the X-ray emission is probably originated from the inner part of the relativistic jet, while, in the SSRQs and in some radiogalaxies the origin of the X-ray emission is probably more similar to radio-quiet AGNs (QSOs and Seyfert galaxies, respectively). The distinction between blazars and non-blazars would require specific data in order to 
Table 2. Optical properties of the radio loud and of the comparison sample.

\begin{tabular}{lcccc}
\hline \hline Sample & Radio loud & \multicolumn{3}{c}{ Comparison } \\
\hline Number of sources & 25 & & 53 & \\
Number of identified sources & 20 & $82 \%$ & 51 & $96 \%$ \\
& & & & \\
Type 1 AGNs & 12 & $60 \%$ & 40 & $78 \%$ \\
Type 2 AGNs & 2 & $10 \%$ & 7 & $14 \%$ \\
Galaxies & 1 & $5 \%$ & 2 & $4 \%$ \\
Clusters of galaxies & 1 & $5 \%$ & 1 & $2 \%$ \\
BL Lacs & 4 & $20 \%$ & 1 & $2 \%$ \\
\hline
\end{tabular}

measure, for instance, the variability and the polarisation that are characteristic features of the blazar class. However, we can make a first attempt at selecting the blazars in the sample by using the existing radio data taken from many different surveys. Therefore, we have derived (non simultaneous) data at different radio frequencies from that of the NVSS (1.4 GHz), and/or radio data with a better resolution (i.e. the data from FIRST survey, made with VLA in B-array) in order to constrain the core-dominance.

\subsection{Radio spectral indices}

Most of the radio fluxes are collected at $5 \mathrm{GHz}$ from the GB6 catalogue (Gregory et al. 1996) while a few objects have been detected at low frequencies in the WENSS survey (Rengelink et al. 1997) or in the TEXAS survey (Douglas et al. 1996). Finally, a few other radio fluxes are taken from the literature. In total, we have fluxes at one or more frequencies other than $1.4 \mathrm{GHz}$ for 15 objects out of 25 . The typical definition of "flat" spectrum radio source usually requires a spectral index flatter than 0.5 (e.g. Brunner et al. 1994). However, when nonsimultaneous data, and/or data taken with instruments with different resolutions, are used, a more correct limit for the distinction between blazars and non-blazars is $\alpha=0.7$ (e.g. Perlman et al. 1998; Cavallotti et al. 2004). By adopting this limit the reliability of the blazar classification is not $100 \%$. However, as discussed in Perlman et al. (1998), this limit, coupled with the $\mathrm{X}$-ray emission, leads to a small fraction $(\sim 5 \%)$ of non-blazars wrongly classified as "blazars". Indeed, we have found one object (XBSJ012654+191246), a brigh ( 1.4 Jy) radiogalaxy, whose high resolution radio map shows a clear extended structure (Owen \& Ledlow 1997) in spite of a relatively flat radio spectral index (0.53). In the following analysis, we consider this object as non-blazar.

\subsection{Radio morphology}

The FIRST data are available for 12 sources out of $25 \mathrm{RL}$ ( 8 of these have also fluxes at other frequencies) and for the 3 sources RQ. From the FIRST survey we have taken a radio map at $1.4 \mathrm{GHz}$ with a resolution (beam size $\sim 5^{\prime \prime}$ ) a factor $\sim 10$ better than that of the NVSS data. We have then calculated a core-dominance parameter based on the peak and on the total flux derived from the FIRST catalogue. The parameter is defined as:

core dominance $=\frac{S_{\text {core }}}{S_{\text {ext }}}$

where we have assumed the core flux density $\left(S_{\text {core }}\right)$ equal to the peak flux density of the brightest component, and the extended flux density $\left(S_{\text {ext }}\right.$ ) equal to the total flux density minus the peak flux density. The total flux density is the integrated flux density taken from the FIRST catalogue. For 3 sources (2 AGNs and 1 unidentified object) the FIRST map shows a double or triple morphology and a core dominance $<0.5-0.6$ (see Fig. 4). These 3 objects are thus considered as "nonblazars", on the basis of the radio morphology. We note that the remaining objects, that are compact at the FIRST resolution, cannot be classified as "blazar" since the beam size is not necessarily small enough to resolve the source, given the observed range of redshift. For this reason, we use the morphological information only to classify the 3 extended objects as "non-blazars".

\subsection{Summary of the radio classification}

In summary, based on the available radio or optical data, we have distinguished the RL AGNs in the sample between "blazars" and "non-blazars" according to the following criteria:

- all the objects optically classified as BL Lacs are considered blazars;

- all the objects with a flat radio spectrum $(\alpha<0.7)$ are classified as blazars while all the sources with a steep radio spectrum ( $\alpha>0.7$ or a lower limit on $\alpha$ above 0.7 ) are classified as non-blazars. As discussed above, there is one exception (XBSJ012654+191246), i.e. a powerful radiogalaxy that has been classified as "non-blazar" on the basis of more detailed data taken from the literature in spite of its relatively flat radio spectrum;

- all the objects with a double or triple radio morphology (based on FIRST data) are classified as non-blazars.

In total, we have divided the sample of radio-loud AGNs into 9 blazars, 10 non-blazars and 6 sources without a radio classification because of the lack of specific data. This classification is reported in Col. 9 of Table 1.

\section{The X-ray spectral analysis}

Thanks to the availability of good XMM-Newton data for the XBSS sample, we can perform a reliable X-ray spectral analysis for each source in the sample. The XBSS sample has been defined using only the data from the MOS2 detector. In order to increase the statistics for the X-ray spectra, the data from the MOS1 and the pn detectors, when available, are also considered and used for the analysis. Table 3 shows the EPIC detectors used for the spectral analysis of each source along with the values of Galactic column densities (Dickey \& Lockman 1990) towards the selected XMM-Newton pointings.

The X-ray spectra usually cover the $0.2-10 \mathrm{keV}$ energy range and have been extracted using a circular region with a 


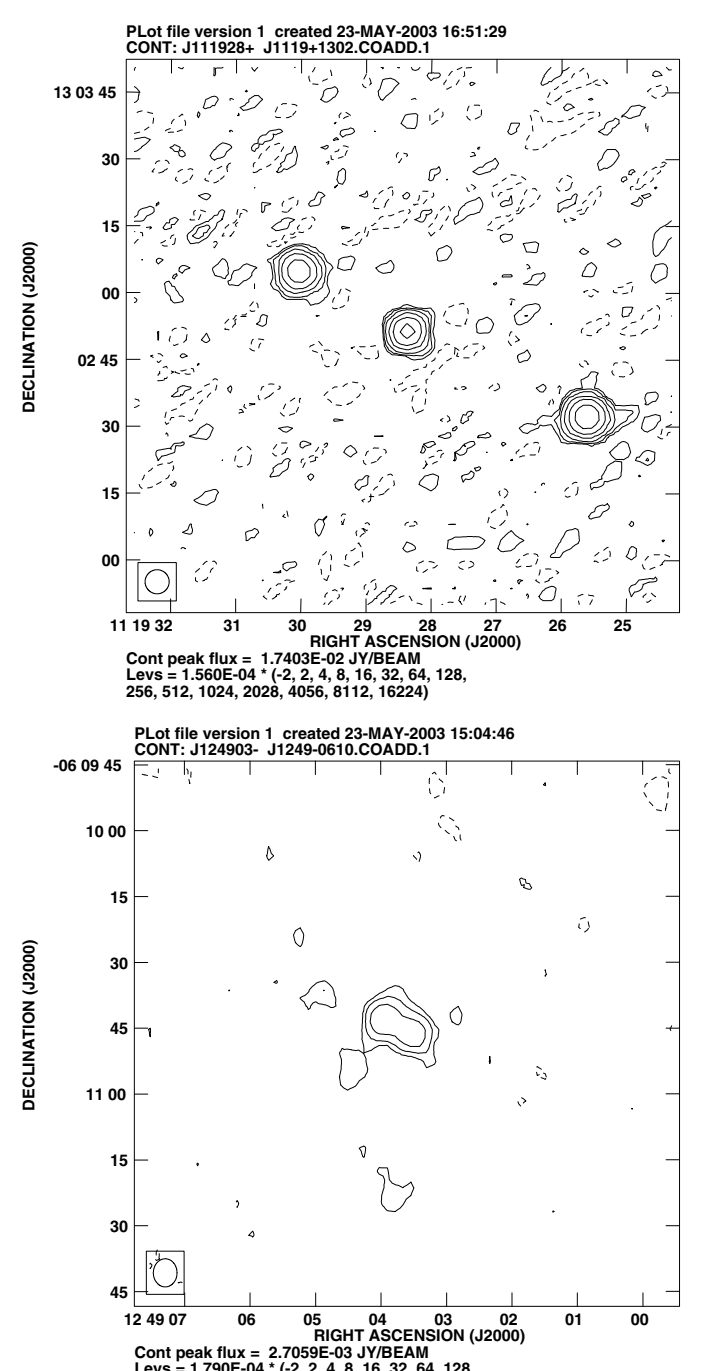
Cont peak flux $=2.7059 \mathrm{E}-\mathrm{-O}$ JY/BEAM
Levs $=1.790 \mathrm{~J}-04 \times(-2,2,4,8,16,32,64,128$,

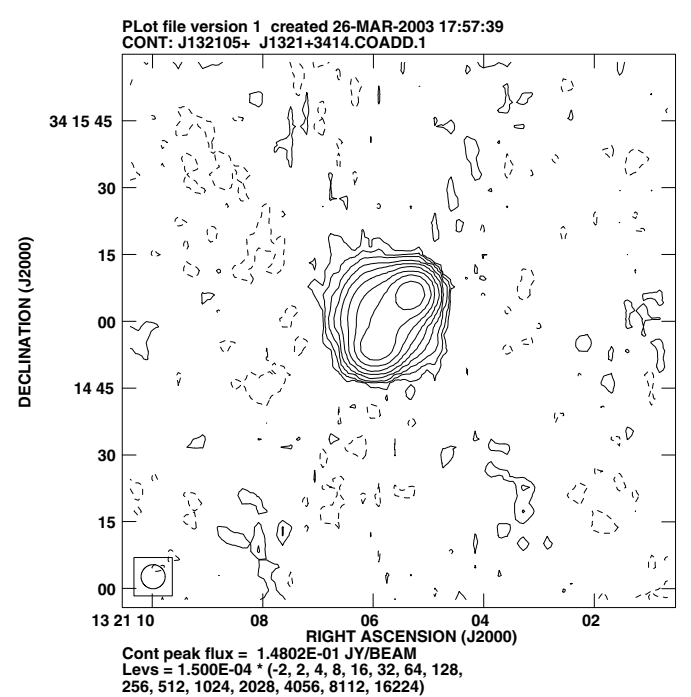

Fig. 4. Radio maps at $1.4 \mathrm{GHz}$ of the $3 \mathrm{RL}$ AGNs resolved in the FIRST maps and showing double or triple morphology. From top to bottom: XBSJ111928.5+130250, XBSJ124903.6-061049 and XBSJ132105.5+341459.

radius of $17.5^{\prime \prime}-25^{\prime \prime}$. The background has been extracted in nearby circular source free regions of an area a factor $\sim 4$ larger than the one used to extract the source counts. In order to improve the statistics the MOS1 and MOS2 counts obtained with the same filters have been combined together. The data of the MOS and of the pn detectors have been binned in order to have at least 10-25 counts for each channel, depending on the brightness of the source. For the analysis of the data we used the XSPEC 11.2.0 software package. We have fitted simultaneously the MOS and pn data, leaving the relative normalization free to vary (range 0.9-1.2). Errors are given at the $90 \%$ confidence level for one interesting parameter $\left(\Delta \chi^{2}=2.71\right)$.

We started the analysis of all the spectra by fitting a simple rest frame absorbed power law model, including the Galactic absorption along the line of sight (derived from Dickey \& Lockman 1990).

For the 2 sources for which the number of counts is not high enough to accurately determine both the $\Gamma$ and the level of absorption, we have fixed the value of $\Gamma$ to 1.9 in order to better constrain the correct value of $N_{\mathrm{H}}$. The value of $\Gamma=1.9$ corresponds to the average value found for the type 1 AGNs in a complete and representative subsample of the "hard" XBSS (Caccianiga et al. 2004). As described in the next section, this value turned out to be slightly lower than the average value derived from the analysis of the RL AGN sample (2.0 \pm 0.4$)$ but fully consistent with it within the errors. These 2 sources are marked with an asterisk in Col. 4 of Table 4.

In total, for 20 sources the absorbed power law model describes correctly the data. In three cases, instead, a soft-excess component, modelled with a black-body spectrum with temperature ranging from 0.15 to $0.23 \mathrm{keV}$ is required in addition to the power-law component. For the remaining 2 sources (XBSJ012654.3+191246 and XBSJ122658.1+333246) the best fit is obtained with a single thermal spectrum. More details on these 2 objects are given below.

XBSJ012654.3+191246. For this source, optically classified as "normal galaxy" (see Fig. 3), the simple absorbed power law model gives a fit with strong residuals and a best-fit slope too steep $(\Gamma=4.6)$. If $\Gamma$ is frozen to the mean value observed in AGNs $(\Gamma=1.9)$ then the fit is unacceptable $\left(\chi^{2} / v=3.62\right.$, with 7 d.o.f.). A model containing only a thermal component (with $k T=0.69 \mathrm{keV}$ ) gives a better fit. In particular, we have used the spectrum from hot diffuse gas based on the model "mekal". As discussed in Sect. 4.1, this is a known radiogalaxy. The X-ray luminosity derived from this source $\left(3.5 \times 10^{40} \mathrm{erg} \mathrm{s}^{-1}\right)$ is within the typical range of X-ray luminosities observed in low-power radiogalaxies (FR I, e.g. Fabbiano et al. 1984 and Fabbiano 1989).

XBSJ122658.1+333246. This source is a high-redshift $(z=$ 0.89 ) cluster of galaxies identified in the WARPS survey (Ebeling et al. 2001). The X-ray spectral analysis of this object shows that the X-ray spectrum is well described by a thermal component (mekal). We obtain a temperature of $k T=$ $10.42_{-1.43}^{+1.19} \mathrm{keV}$, which is in good agreement with that obtained by Cagnoni et al. (2001) $\left(k T=10.47_{-3}^{+4} \mathrm{keV}\right)$ with Chandra data and with that obtained from the Sunyaev-Zeldovich measurement by Joy et al. (2001) $\left(k T=10.00_{-1.5}^{+2.0} \mathrm{keV}\right)$. This is one of the highest temperatures ever measured in a cluster of galaxies. In conclusion, in this object the X-ray emission is 
Table 3. The XMM-Newton detectors used for the spectral analysis of the RL sample.

\begin{tabular}{|c|c|c|c|c|}
\hline $\begin{array}{l}\text { Name } \\
(1)\end{array}$ & $\begin{array}{l}\text { Obs ID } \\
\text { (2) }\end{array}$ & $\begin{array}{l}\text { Gal } N_{\mathrm{H}}\left(\times 10^{20}\right) \\
\text { (3) }\end{array}$ & $\begin{array}{l}\text { Detector } \\
\text { (4) }\end{array}$ & $\begin{array}{c}\text { Notes } \\
(5)\end{array}$ \\
\hline XBSJ000100.2-250501 & 0125310101 & 1.88 & MOS2, MOS1, pn & \\
\hline XBSJ003255.9+394619 & 0065770101 & 6.57 & MOS2, MOS1, pn & \\
\hline XBSJ012505.4+014624 & 0109860101 & 3.10 & MOS2, MOS1, pn & \\
\hline XBSJ012654.3+191246 & 0112600601 & 4.80 & MOS2, MOS1 & $\mathrm{a}$ \\
\hline XBSJ013240.1-133307 & 0084230301 & 1.64 & MOS2, MOS1, pn & \\
\hline XBSJ013811.7-175416 & 0111430101 & 1.44 & MOS2, MOS1 & $\mathrm{a}$ \\
\hline XBSJ021640.7-044404 & 0112371701 & 2.42 & MOS2, MOS1, pn & \\
\hline XBSJ033226.9-274107 & 0108060501 & 0.90 & MOS2, MOS1, pn & \\
\hline XBSJ052108.5-251913 & 0085640101 & 1.92 & MOS2, MOS1, pn & $\mathrm{b}$ \\
\hline XBSJ061342.7+710725 & 0009220601 & 8.38 & MOS2, MOS1, pn & \\
\hline XBSJ084026.2+650638 & 0111400101 & 4.29 & MOS2, MOS1 & \\
\hline XBSJ101511.8+520708 & 0086750101 & 0.76 & MOS2, MOS1, pn & $\mathrm{b}$ \\
\hline XBSJ101706.5+520245 & 0086750101 & 0.76 & MOS2, MOS1, pn & \\
\hline XBSJ102016.1+082143 & 0093640301 & 2.99 & MOS2, MOS1, pn & \\
\hline XBSJ111928.5+130250 & 0093641101 & 2.43 & MOS2, MOS1 & $\mathrm{c}$ \\
\hline XBSJ122658.1+333246 & 0070340501 & 1.39 & MOS2, MOS1, pn & $\mathrm{b}$ \\
\hline XBSJ123538.6+621644 & 0111550101 & 1.49 & MOS2, MOS1, pn & \\
\hline XBSJ124903.6-061049 & 0060370201 & 2.13 & MOS2, MOS1, pn & \\
\hline XBSJ132105.5+341459 & 0093640401 & 1.00 & MOS2, MOS1, pn & $\mathrm{b}$ \\
\hline XBSJ133026.6+241520 & 0100240201 & 1.16 & MOS2, MOS1, pn & \\
\hline XBSJ133232.6+111220 & 0061940101 & 1.93 & MOS2, MOS1, pn & \\
\hline XBSJ153452.3+013104 & 0112190401 & 4.89 & MOS2, MOS1, pn & \\
\hline XBSJ164237.9+030014 & 0067340501 & 5.47 & MOS2, MOS1, pn & $\mathrm{b}$ \\
\hline XBSJ221951.6+120123 & 0103861201 & 5.41 & $\operatorname{MOS} 2, \operatorname{MOS} 1, \mathrm{pn}$ & \\
\hline XBSJ235036.9+362204 & 0100241001 & 8.09 & $\operatorname{MOS} 2, \operatorname{MOS} 1, \mathrm{pn}$ & $\mathrm{b}$ \\
\hline
\end{tabular}

Column 1: source name;

Col. 2: observation ID;

Col. 3: Galactic column density $\left[\mathrm{cm}^{-2}\right]$;

Col. 4: EPIC cameras used for the spectral analysis;

Col. 5: notes: $\mathrm{a}=\mathrm{pn}$ in small window; $\mathrm{b}=$ source on a gap in the $\mathrm{pn} ; \mathrm{c}=$ source outside the $\mathrm{pn}$.

associated to the hot intra-cluster medium while the radio and the optical emissions are relative to a radiogalaxy that belongs to the cluster.

The results of the X-ray spectral analysis of the radio loud sources are reported in Table 4 and the X-ray spectra are reported in appendix. For one source (XBSJ013240.1-133307), which is optically classified as type 2 AGN, the derived hydrogen column density is significantly larger than the Galactic column density thus confirming the presence of absorption also in the X-ray band. Given its X-ray luminosity $\left(1.5 \times 10^{44} \mathrm{erg} \mathrm{s}^{-1}\right)$ and radio power $\left(6 \times 10^{24} \mathrm{~W} \mathrm{~Hz}^{-1}\right)$, this source can be classified as radio-loud type 2 QSO (see the discussion in Della Ceca et al. 2003 on a similar object discovered with ASCA). We note that for the other type 2 AGN present in the sample (XBSJ221951.6+120123) the X-ray analysis does not reveal a significant absorption $\left(N_{\mathrm{H}} \leq 3.5 \times 10^{21} \mathrm{~cm}^{-2}\right)$. However, the very flat best-fit photon index $(\Gamma=1.39)$ could be suggestive of the presence of a very large absorption plus a flat reprocessed component. Therefore in this source the presence of a large absorption cannot be completely ruled out. Given the large X-ray luminosity, this source is another type 2 radio-loud QSO candidate.

The distribution of the intrinsic $N_{\mathrm{H}}$ derived from the spectral fit is shown in Fig. 5 while the distribution of the photon indices $\Gamma$ is shown in Fig. 6a. The latter histogram does not include the objects for which $\Gamma$ has been fixed to 1.9. The mean value is 2.0 with a standard deviation of 0.4 .

\section{Comparison between RL and RQ AGNs}

\subsection{The comparison sample}

The $25 \mathrm{X}$-ray sources studied in this work are radio loud AGNs. In order to investigate the X-ray spectral differences between RL and RQ AGNs, we have defined a comparison sample. This sample is selected from the same XBSS catalogue and it consists of X-ray extragalactic sources which are not detected in the NVSS survey, thus having a flux density below $2.5 \mathrm{mJy} / \mathrm{beam}$. 
Table 4. Best-fit parameters for the X-ray spectral analysis.

\begin{tabular}{|c|c|c|c|c|c|c|c|c|}
\hline $\begin{array}{l}\text { name } \\
\text { (1) }\end{array}$ & $\begin{array}{l}\text { id } \\
(2)\end{array}$ & $\begin{array}{l}\text { Model } \\
\text { (3) }\end{array}$ & $\begin{array}{r}\Gamma \\
(4)\end{array}$ & $\begin{array}{r}N_{\mathrm{H}} \\
(5) \\
\left(\times 10^{22}\right)\end{array}$ & $\begin{array}{l}k T \\
(6)\end{array}$ & $\begin{array}{l}\chi_{v}^{2} / \text { d.o.f. } \\
\text { (7) }\end{array}$ & $\begin{array}{r}f_{2-10 \mathrm{keV}} \\
(8) \\
\left(\times 10^{-13}\right)\end{array}$ & $\begin{array}{r}\log \left(L_{2-10 \mathrm{keV}}\right) \\
(9)\end{array}$ \\
\hline XBSJ000100.2-250501 & AGN1 & $\mathrm{PL}$ & $1.68_{-0.2}^{+0.2}$ & $0.72_{-0.29}^{+0.23}$ & - & $1.19 / 40$ & 1.39 & 44.67 \\
\hline XBSJ003255.9+394619 & AGN1 & PL & $2.11_{-0.41}^{+0.92}$ & $<0.42$ & - & $0.43 / 3$ & 0.44 & 44.62 \\
\hline XBSJ012505.4+014624 & AGN1 & PL & $1.65_{-0.06}^{+0.06}$ & $<0.47$ & - & $0.86 / 101$ & 1.62 & 45.33 \\
\hline XBSJ012654.3+191246 & G & $\mathrm{T}$ & - & $0.69_{-0.00}^{+8.24}$ & $0.63_{-0.18}^{+0.02}$ & $0.63 / 6$ & 0.06 & 40.55 \\
\hline XBSJ013240.1-133307 $7^{(1)}$ & AGN2 & PL & $1.9 *$ & $2.54_{-0.56}^{+0.71}$ & - & $1.11 / 19$ & 1.71 & 44.41 \\
\hline XBSJ013811.7-175416 & BL? & PL & $2.47_{-0.21}^{+0.25}$ & $0.07_{-0.06}^{+0.06}$ & - & $1.40 / 31$ & 0.36 & 43.68 \\
\hline XBSJ021640.7-044404 ${ }^{(2)}$ & AGN1 & $\mathrm{PL}+\mathrm{BB}$ & $2.28_{-0.26}^{+0.31}$ & $0.56_{-0.56}^{+0.28}$ & $0.15_{-0.13}^{+0.00}$ & $1.08 / 93$ & 1.14 & 44.73 \\
\hline XBSJ033226.9-274107 & AGN1 & $\mathrm{PL}+\mathrm{BB}$ & $2.04_{-0.15}^{+0.13}$ & $<0.45$ & $0.15_{-0.06}^{+0.06}$ & $0.98 / 95$ & 0.67 & 44.34 \\
\hline XBSJ052108.5-251913 & AGN1 & $\mathrm{PL}+\mathrm{BB}$ & $1.72_{-0.19}^{+0.29}$ & $0.10_{-0.10}^{+1.80}$ & $0.23_{-0.04}^{+0.07}$ & $0.90 / 61$ & 2.46 & 45.26 \\
\hline XBSJ061342.7+710725 & $\mathrm{BL}$ & PL & $2.67_{-0.15}^{+0.17}$ & $0.14_{-0.32}^{+0.05}$ & - & $0.79 / 69$ & 1.87 & 43.75 \\
\hline XBSJ084026.2+650638 & AGN1 & PL & $1.71_{-0.18}^{+0.19}$ & $0.29_{-0.2}^{+0.03}$ & - & $1.08 / 34$ & 1.15 & 44.89 \\
\hline XBSJ101511.8+520708 & AGN1 & PL & $2.06_{-0.31}^{+0.47}$ & $<0.18$ & - & $1.07 / 17$ & 0.76 & 45.55 \\
\hline XBSJ101706.5+520245 & BL & PL & $2.82_{-0.44}^{+0.83}$ & $0.006_{-0.001}^{+0.008}$ & - & $1.06 / 14$ & 0.32 & 43.53 \\
\hline XBSJ102016.1+082143 & - & PL & $1.97_{-0.23}^{+0.47}$ & $<6.89 \mathrm{E}-2$ & - & $0.40 / 17$ & 0.67 & - \\
\hline XBSJ111928.5+130250 & AGN1 & PL & $1.90_{-0.17}^{+0.33}$ & $<0.62$ & - & $0.95 / 6$ & 1.11 & 45.74 \\
\hline XBSJ122658.1+333246 & $\mathrm{CL}$ & $\mathrm{T}$ & - & $<0.01$ & $10.42_{-1.43}^{+1.19}$ & $0.93 / 205$ & 3.06 & 45.14 \\
\hline XBSJ123538.6+621644 & AGN1 & PL & $1.96_{-0.05}^{+0.08}$ & $<0.03$ & - & $0.92 / 112$ & 1.04 & 44.43 \\
\hline XBSJ124903.6-061049 & AGN1 & PL & $2.12_{-0.04}^{+0.18}$ & $<0.03$ & - & $1.20 / 70$ & 0.87 & 44.35 \\
\hline XBSJ132105.5+341459 & - & PL & $1.94_{-0.17}^{+0.15}$ & $0.03_{-0.03}^{+0.03}$ & - & $1.18 / 53$ & 1.64 & - \\
\hline XBSJ133026.6+241520 & - & PL & $2.74_{-0.04}^{+0.06}$ & $<0.004$ & - & $0.85 / 132$ & 0.42 & - \\
\hline XBSJ133232.6+111220 & - & PL & $1.9 *$ & $<0.12$ & - & $1.42 / 3$ & 0.58 & - \\
\hline XBSJ153452.3+013104 & AGN1 & PL & $1.75_{-0.04}^{+0.09}$ & $<0.05$ & - & $0.98 / 112$ & 7.42 & 45.95 \\
\hline XBSJ164237.9+030014 & - & PL & $1.62_{-0.14}^{+0.51}$ & $<0.08$ & - & $1.37 / 7$ & 0.90 & - \\
\hline XBSJ221951.6+120123 & AGN2 & PL & $1.39_{-0.02}^{+0.36}$ & $<0.35$ & - & $1.26 / 12$ & 1.26 & 44.10 \\
\hline XBSJ235036.9+362204 & $\mathrm{BL}$ & PL & $1.90_{-0.22}^{+0.34}$ & $0.03_{-0.03}^{+0.17}$ & - & $1.08 / 13$ & 2.06 & 43.87 \\
\hline
\end{tabular}

Column 1: source name; ${ }^{(1)}$ The best fit values for this source are taken from Caccianiga et al. (2004); ${ }^{(2)}$ This source belongs also to the HBS28 sample discussed in Caccianiga et al. (2004). In the X-ray analysis presented here, however, we have added a soft excess component and the best fit parameters (the $N_{\mathrm{H}}$ in particular) are newly calculated;

Col. 2: optical classification;

Col. 3: best-fit model $(\mathrm{PL}=$ absorbed power law; $\mathrm{T}=$ thermal component $($ mekal); $\mathrm{PL}+\mathrm{BB}=$ absorbed power law plus soft excess $(\mathrm{modelled}$ with a black-body spectrum);

Col. 4: best-fit photon index of the power-law component (* in this case the value of the photon index has been fixed to 1.9);

Col. 5: best fit intrinsic hydrogen column density $\left[\mathrm{cm}^{-2}\right]$;

Col. 6: temperature of the thermal component or of the soft excess [keV];

Col. 7: reduced Chi squared and degrees of freedom of the best fit;

Col. 8: observed 2-10 keV flux (corrected for the Galactic absorption) $\left[\mathrm{erg}^{-2} \mathrm{~cm}^{-1}\right.$;

Col. 9: intrinsic luminosity in the $2-10 \mathrm{keV}$ energy range [erg s${ }^{-1}$ ].

In order to have a high number of sources spectroscopically identified, we have considered only the area of the sky with declination $>-30^{\circ}$ and with right ascension $20^{\mathrm{h}}<\alpha<2^{\mathrm{h}}$. In this area there are 67 sources out of which 65 have a spectroscopic classification. For the comparison we use only the extragalactic subsample composed of 51 objects plus the 2 unidentified sources. On the basis of their hardness-ratios and X-ray-to-optical flux ratios, in fact, these 2 sources are very likely to be extragalactic objects (see Della Ceca et al. 2004). In conclusion, the comparison sample contains 53 XBSS sources with a radio flux density at $1.4 \mathrm{GHz}$ below $2.5 \mathrm{mJy}$.
The classification breakdown of this comparison sample is presented in Table 2. Apart from one cluster of galaxies and 2 "normal" galaxies, the remaining 48 objects are optically classified as AGNs. From the X-ray spectral analysis we have direct evidence of the presence of an AGN also in the two objects optically classified as "normal" galaxies. The comparison sample is thus mainly composed by AGNs.

For a reliable analysis of the differences between RQ and RL AGNs it is important that the comparison sample is representative of the RQ population. Given the lack of measured radio fluxes, for the sources in the comparison sample it is only 


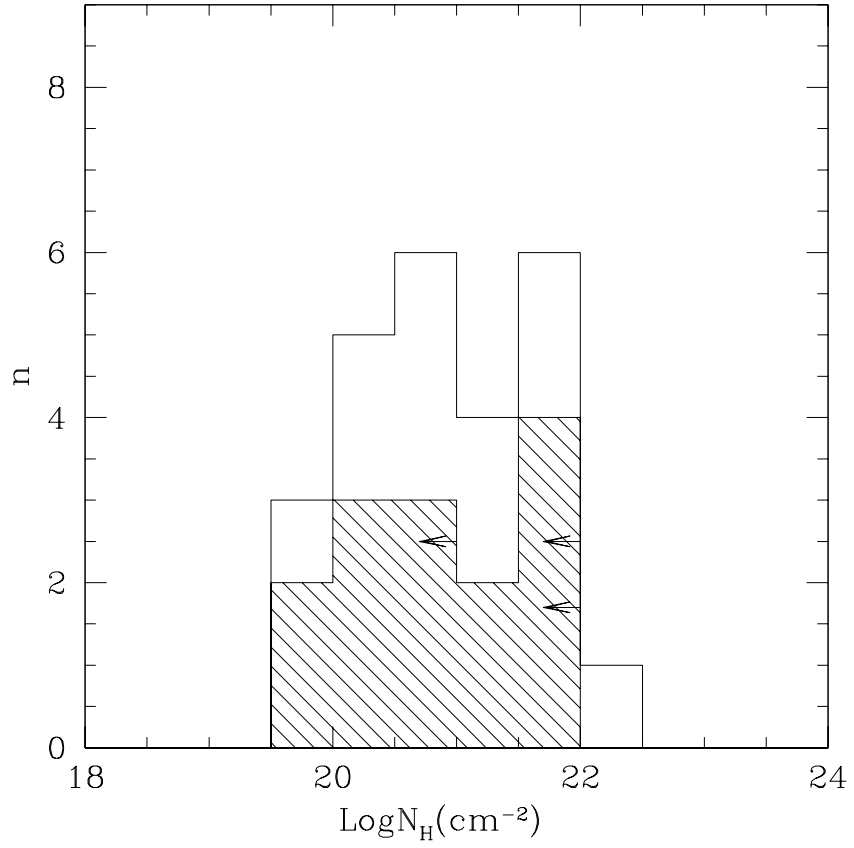

Fig. 5. The distribution of the intrinsic $N_{\mathrm{H}}$ derived from the spectral fit. The shaded histogram shows the upper limits on $N_{\mathrm{H}}$ while the empty histogram represents the detections.

possible to compute an upper limit on the radio-loudness parameter (see Sect. 2.1). In about $30 \%$ of the sources the computed upper limit is below the dividing line between RQ and RL AGNs so that they can be classified as RQ sources. For the remaining objects a firm classification as RQ objects is not possible with the present data. However, the analysis described in Sect. 2 of the distribution of the radio-loudness parameter (and the upper limits) indicates that among the sources not detected in the NVSS we expect a small contamination of RL AGNs (about 2 objects in the comparison sample defined here). Hence, even if these few RL AGNs cannot be identified without a deeper radio follow-up, we can consider the whole comparison sample as statistically representative of the RQ AGN population.

The X-ray spectra of the 53 objects contained in the comparison sample have been extracted and analysed with the same procedures and criteria described previously for the sample of RL AGNs.

In Fig. 7 we have reported the distribution of the deabsorbed X-ray luminosities $(2-10 \mathrm{keV})$ of the two samples as resulting from the X-ray spectral analysis. Although the RL AGNs have on average slightly higher luminosities (mean $L_{\mathrm{X}}=10^{44.4} \mathrm{erg} \mathrm{s}^{-1}$ ) than RQ AGNs (mean $L_{\mathrm{X}}=10^{44.0} \mathrm{erg} \mathrm{s}^{-1}$ ), the two distributions cover a very similar range of values and the KS probability $\left(P_{\mathrm{KS}}=6 \%\right)$ is only marginally indicative that the two distributions are not derived from the same parent distribution. For this reason, we consider the comparison sample as a good choice to match the X-ray properties of RL and RQ AGNs since they both contain objects within a similar range of luminosities.
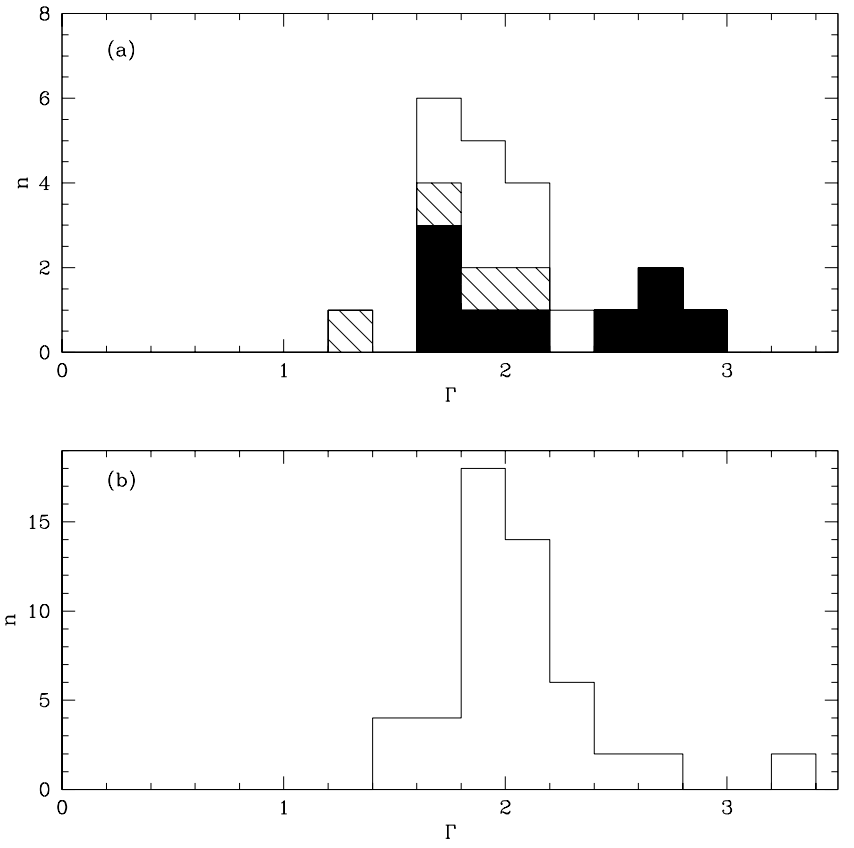

Fig. 6. The X-ray photon index distribution obtained from the spectral fitting for the RL sources a) and for the comparison sample b). In a) the black histogram represents the blazar, the empty histogram represents the non-blazar sources and the shaded histogram shows the sources with no data to allow a radio classification. In these histograms the sources for which the value of $\Gamma$ has been fixed to 1.9 (see text for details) and those fitted only by a thermal emission are not included.

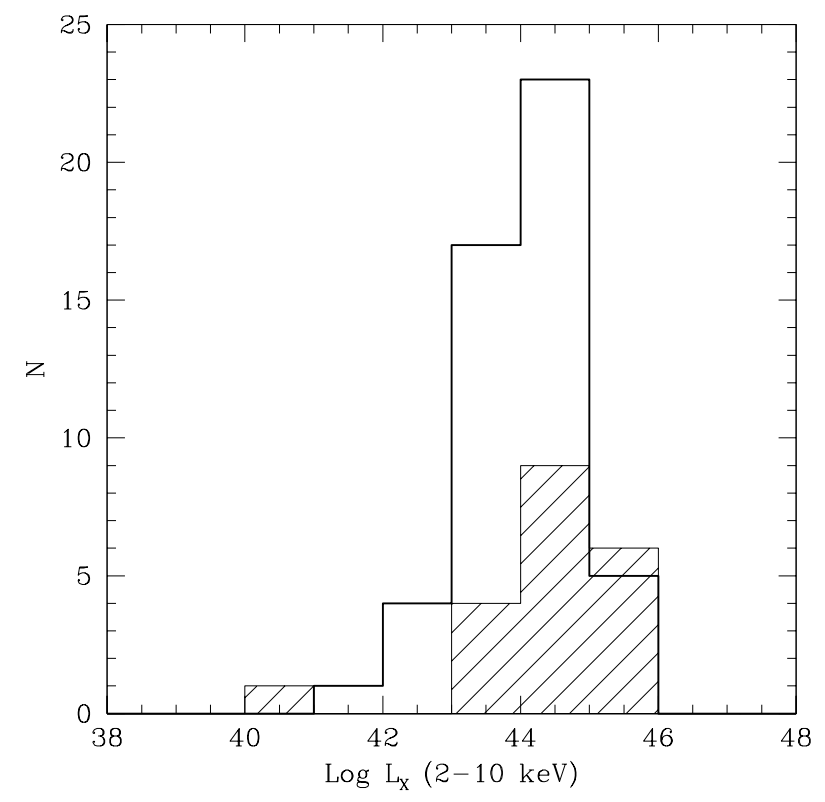

Fig. 7. Distribution of the de-absorbed $2-10 \mathrm{keV}$ luminosity of the sample of RL AGNs (shaded histogram) and the comparison sample (thick line). Only the sources in the 2 samples with a measured redshift are considered.

\subsection{Differences in the optical composition}

In Table 2 the optical compositions of the radio-loud and of the comparison samples are summarized and compared. The main difference between the two samples rests in the percentage of 
BL Lac objects which is low (2\%) in the comparison sample and much higher $(20 \%)$ in the sample of RL AGNs. We recall here that the only BL Lac found in the comparison sample is not necessarily a RQ object since, as stated above, we expect a small percentage ( $\sim 2$ objects) of RL AGNs also in this sample. Thus, the percentage of BL Lacs in the 2 samples could be even more different than what is reported in Table 2 . This result confirms very clearly that BL Lac objects (at least, the X-ray selected ones) are radio-loud AGNs, as firstly pointed out by Stocke et al. (1990). Hence, if radio-quiet BL Lacs exist, as recently suggested by Londish et al. (2004), these objects must be also "X-ray-quiet", i.e. they must have a very anomalous Spectral Energy Distribution (SED).

If we exclude the BL Lac objects from the 2 samples and re-normalize the percentages of the different classes of sources, the relative numbers are very similar in the two samples (the number of clusters and normal galaxies is too small to have any statistical significance). We conclude that the requirement of a radio emission does not change significantly the composition of the sample, apart from the BL Lac objects that are greatly favoured by the radio constraint. Indeed, the combination of X-ray and radio data has been widely used in the last years to select large samples of BL Lacs (Maccacaro et al. 1998; Laurent-Muehleisen et al. 1998; Perlman et al. 1998; Caccianiga et al. 1999; Giommi et al. 1999).

\subsection{Comparing the intrinsic $X$-ray spectral index}

We have then compared the X-ray photon indices of the power law component in the two samples. The comparison of the spectral index gives in fact important pieces of information on the similarities/differences of the primary source of the X-ray emission.

In the following analysis we have not included the sources for which the value of $\Gamma$ has been fixed to 1.9 and those fitted only by a thermal spectrum. Figure 6 shows the distribution of the best-fit spectral indices. The mean value of $\Gamma$ is very similar in the two samples $\left(\Gamma_{\mathrm{RL}}=2.0\right.$ and $\Gamma_{\mathrm{RQ}}=2.1$ respectively $)$.

We have then computed the intrinsic dispersion of the values of $\Gamma$ following the method described in Maccacaro et al. (1988) which takes into account the errors on the values of $\Gamma$ (under the assumption of a Gaussian distribution). In Fig. 8 the confidence contours $(68 \%, 90 \%$, and $95 \%)$ for the joint determination of $\langle\Gamma\rangle$ and of the intrinsic dispersion $\sigma$ are presented. It is evident that, while the mean values of $\Gamma$ in the two samples are very similar, the intrinsic spreads are significantly $(95 \%)$ different $\left(\sigma_{\mathrm{RL}}=0.34\right.$ vs. $\left.\sigma_{\mathrm{RQ}}=0.18\right)$.

We have analysed the possibility that the observed broader distribution is due to the presence, in the RL AGN sample, of the blazars. In fact, observing the distribution presented in Fig. 6a, BL Lacs and the FSRQs are populating respectively the steep and flat tails of the distribution $\left(\langle\Gamma\rangle_{\mathrm{BL} \text { Lac }}=2.5\right.$ and $\langle\Gamma\rangle_{\mathrm{FSRQ}}=2.0$ ). We have thus re-analysed the two samples considering separately the non-blazars and the blazars (see Fig. 9). Although the number of objects is small, it is clear that in the first case the two distributions are fully consistent, both in terms of $\langle\Gamma\rangle$ and in terms of intrinsic spread $\left(\sigma_{\mathrm{RL}}=0.12\right.$

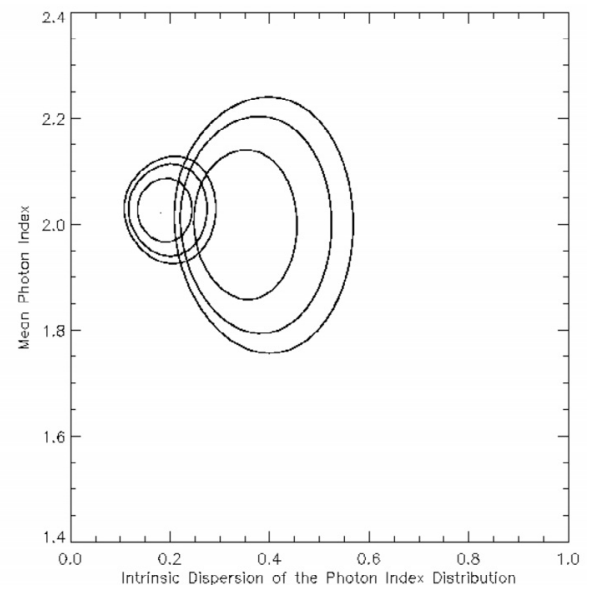

Fig. 8. Confidence contours $(68 \%, 90 \%$, and $95 \%)$ for the joint determination of the $\langle\Gamma\rangle$ and the intrinsic dispersion $\sigma$ of the photon index distribution for the RL AGNs (curves on the right) and RQ AGNs (curves on the left). In the analysis we have not included the sources with a fixed value of $\Gamma$ and those fitted only by a thermal emission.

and $\left.\sigma_{\mathrm{RQ}}=0.18\right)$ while, in the case of blazars, the intrinsic spread is significantly larger $\left(\sigma_{\text {blazar }}=0.44\right)$ than the one observed in RQ AGNs.

Overall, the results discussed here show that the distribution of $\Gamma$ of the non-blazars is very similar, in terms of the mean value and the intrinsic spread, to the one observed in RQ AGNs. The class of blazars, instead, presents a much broader distribution of values of $\Gamma$ (although the mean value is consistent with that observed in the comparison sample) with the FSRQ and the BL Lac objects populating respectively the flat and the steep tails of the distribution. Therefore, the presence of a significant number of blazars in the RL sample broadens the observed distribution of $\Gamma$ but it does not change the mean value. In the next section we further investigate the origin of the X-ray emission of the blazars present in the sample of RL AGNs.

\section{The X-ray spectra of blazars}

According to the beaming model (e.g. Urry \& Padovani 1995, and references therein) the class of blazars is represented by RL AGNs observed very close to the direction of the relativistic jets. The non-thermal emission produced within the jet is thus relativistically amplified towards the observer becoming dominant in most of the observing frequencies. The SED of blazars is usually modelled with two large humps produced, respectively, via synchrotron and Inverse Compton (IC) emission. The frequencies at which these two components peak can vary in a wide range of values leading to very different radio-tooptical and optical-to-X-ray ratios: very large values of the synchrotron peak frequency $\left(v>10^{15.5} \mathrm{~Hz}\right)$ typically produce objects with very flat X-ray-to-radio spectral indices ${ }^{3}\left(\alpha_{\mathrm{RX}}<0.7\right)$ while low values of the peak frequencies $\left(v<10^{15.5} \mathrm{~Hz}\right)$ produce steep $\alpha_{\mathrm{RX}}\left(\alpha_{\mathrm{RX}}>0.7\right)$.

\footnotetext{
3 The two-point spectral index $\alpha_{\mathrm{RX}}$ is defined in the following way: $\alpha_{\mathrm{RX}}=-\log \left(S_{\mathrm{R}} / S_{\mathrm{X}}\right) / \log \left(\nu_{\mathrm{R}} / \nu_{\mathrm{X}}\right)$, where $S_{\mathrm{R}}, \nu_{\mathrm{R}}$ and $S_{\mathrm{X}}, v_{\mathrm{X}}$ are the monochromatic fluxes and frequencies in the radio $(5 \mathrm{GHz})$ and in the $\mathrm{X}$-rays (2 keV), respectively.
} 

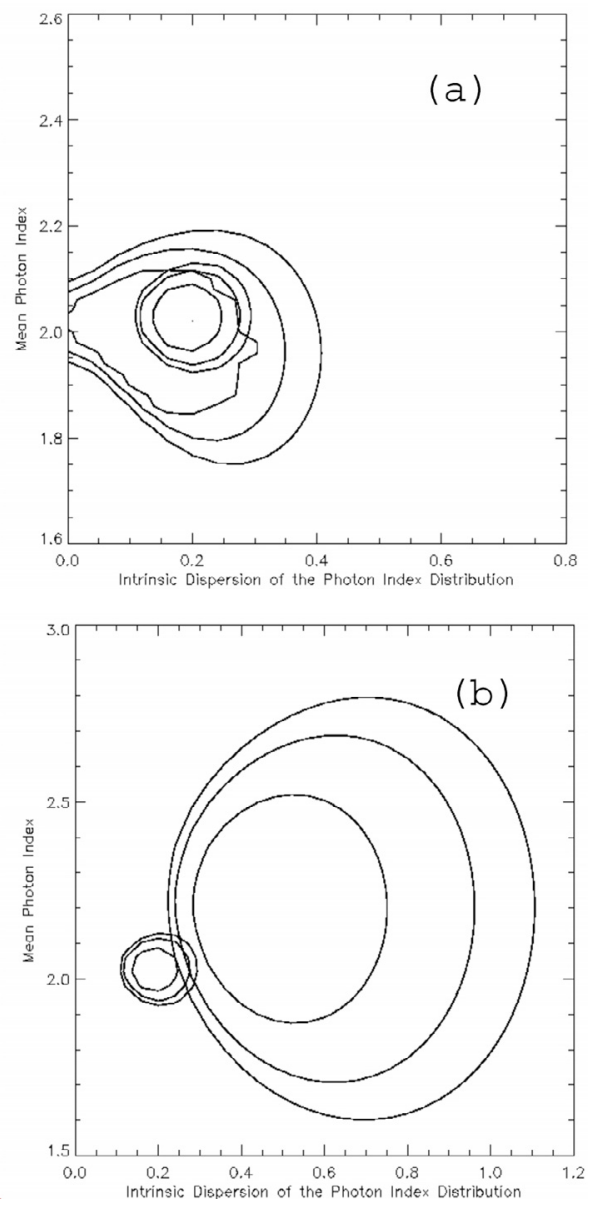

Fig. 9. The same as in Fig. 8 but keeping separate the non-blazars (panel a)) and the blazars (panel b)).

The first systematic ROSAT observations of samples of blazars have revealed a connection between the X-ray spectral index and the value of $\alpha_{\mathrm{RX}}$ : objects with $\alpha_{\mathrm{RX}}<0.8$ show steep values of $\Gamma(\sim 2.5)$ while blazars with $\alpha_{\mathrm{RX}}>0.8$ have flat X-ray spectral indices $(\langle\Gamma\rangle \sim 1.5-2.0$; Padovani \& Giommi 1996; Padovani et al. 1997; Urry et al. 1996; Lamer et al. 1996). The connection between $\Gamma$ and $\alpha_{\mathrm{RX}}$ (or between $\Gamma$ and the synchrotron peak frequency if a SED is available) has been found also using the data from BeppoSAX and ASCA (Wolter et al. 1998; Padovani et al. 2001; Donato et al. 2001; Beckmann et al. 2002; Padovani et al. 2004).

The usual interpretation of this trend is that the X-ray spectral index must be affected by the energy at which the synchrotron component peaks: for large peak frequencies, the X-ray spectrum includes the (steep) descending part of the synchrotron emission while for low peak frequencies the X-ray spectrum includes the rising (and thus flatter) IC component or a mixture of IC plus synchrotron emission.

In this framework it is interesting to see whether the 9 blazars selected in the sample follow this trend: in this case, in fact, we would have a more direct evidence that the X-ray emission in these sources is linked to the radio emission and is thus produced within the jet.

In Fig. 10a we plot the best-fit photon indices of the 9 blazars versus the $\alpha_{\mathrm{RX}}$ values. Similarly to what is found
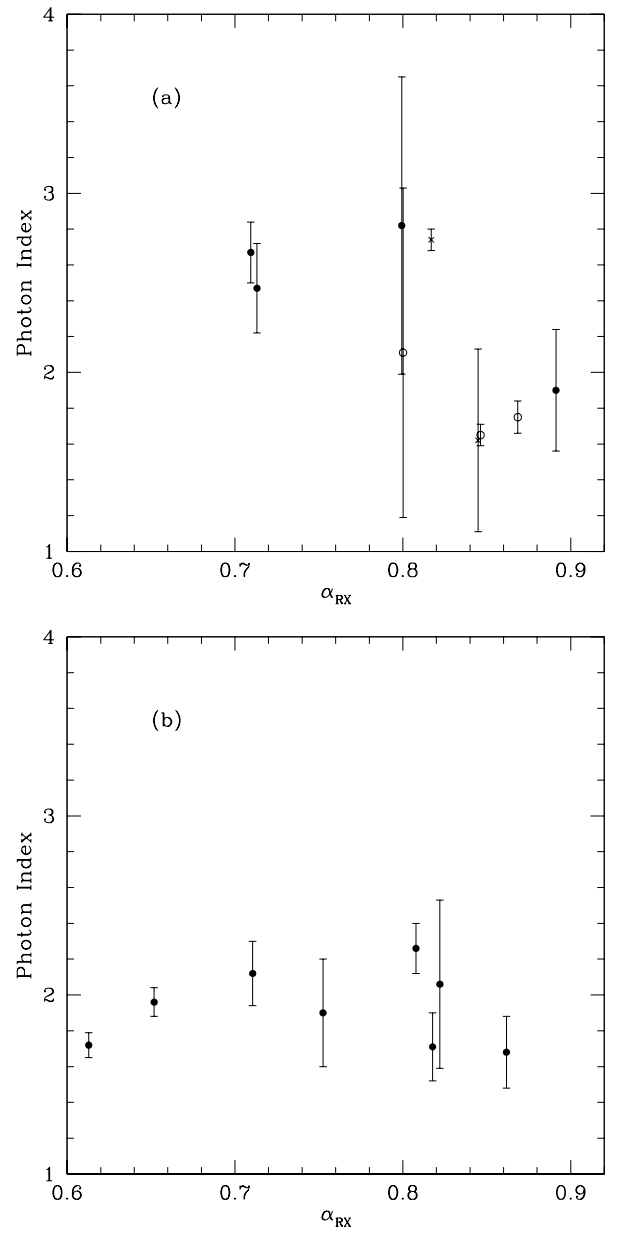

Fig. 10. The X-ray photon index versus the radio-to-X-ray spectral in$\operatorname{dex}\left(\alpha_{\mathrm{RX}}\right)$ for the sample of blazars (panel a)) and the "non-blazars" (panel b)).

in other blazar samples, the objects characterized by low values of $\alpha_{\mathrm{RX}}(<0.85)$ have also the steepest X-ray spectra $(\Gamma \sim$ 2.5-2.8) while all the blazars with large values of $\alpha_{\mathrm{RX}}(>0.85)$ have a photon index below 2 .

This trend is not found for the "non-blazars" (Fig. 10b). In this case, the lack of trend is suggestive that the X-ray emission is not directly related to the radio power, i.e. to the jet emission. This result is in agreement with the idea that, in the "non-blazars", the relativistic beaming is not playing an important role so that the jet contribution to the observed X-ray emission is probably negligible and therefore the X-ray emission is more similar to that observed in RQ AGNs.

\section{Summary and conclusions}

We have presented the analysis of a sample of RL AGNs extracted from the XBSS survey. The NVSS data have been used to select 28 sources of the sample that are also radio emitting at $1.4 \mathrm{GHz}$ with a flux larger than $2.5 \mathrm{mJy}$. Out of these 28 radio/X-ray matches, 25 are classified as radio-loud AGNs on the basis of the radio-loudness parameter $(R>10)$. The presence of an AGN is detected in the optical and/or in the $\mathrm{X}$-ray spectrum for 23 out of the 25 sources. In the remaining 2 sources, optically classified as "galaxy" and "cluster" 
respectively, and for which the X-ray spectrum is modelled by a thermal emission, the presence of an AGN (a radiogalaxy) is strongly suggested by the radio properties (radio-to-optical flux, the radio power or morphology). Therefore, the sample studied here is fully composed by AGN.

Taking into account the flux limit of the NVSS (2.5 mJy/beam), we have estimated that the RL AGNs represent $13 \%$ of the X-ray selected AGNs at a flux limit of $\sim 7 \times$ $10^{-14} \mathrm{erg} \mathrm{s}^{-1} \mathrm{~cm}^{-2}$. We have then selected from the same XBSS a "comparison" sample of 53 sources not detected in the NVSS survey, i.e. with a radio flux density below $2.5 \mathrm{mJy} / \mathrm{beam}$. We have estimated that the large majority of the sources in this sample is represented by RQ AGNs and that at most 2 objects could be RL objects.

The X-ray spectra of the sources in both samples have been extracted, studied and compared.

The main results can be summarized as follows:

- The spectra of the majority of the sources in both samples $(\geq 90 \%)$ are well described by a single power-law. The mean spectral index is similar in both samples $(\Gamma \sim 2)$.

- Although the mean value of $\Gamma$ is similar, the intrinsic spread of the values of $\Gamma$ (i.e. the one computed taking into account the uncertainties) is significantly different in the two samples, with the RL AGNs having a broader distribution ( $\sigma=0.34)$ when compared to the RQ AGNs $(\sigma=0.18)$.

- When blazars (BL Lacs and FSRQs) and "non-blazars" (radiogalaxies and SSRQs) in the RL AGN sample are analysed separately, the larger spread of the spectral index is observed only in the blazars sample while the "non-blazars" show a distribution fully consistent with the one observed in the RQ AGNs. In particular, BL Lacs and FSRQ are populating respectively the steep and the flat tails of the distribution of $\Gamma$.

- The values of the X-ray spectral index of blazars are correlated with the type of SED, i.e. with the radio-to-X-ray spectral index $\left(\alpha_{\mathrm{RX}}\right)$. Such a correlation is not observed in the RL AGNs classified as "non-blazars". This result suggests that the X-ray emission in the blazar class is directly linked, as expected, to the jet emission while this is probably not the case for the "non-blazars", i.e. those RL AGNs whose relativistic jet is not oriented towards the observer. In these objects the relativistic beaming is not important and the observed X-ray emission is likely to have the same origin as in the RQ AGNs.

In conclusion, the analysis presented here has shown that the distribution of the X-ray spectral indices observed in a sample of RL AGNs strongly depends on the importance of relativistic beaming in the selected sources, i.e. on the type of RL AGNs included in the sample under analysis. First, the inclusion of a significant fraction of "oriented" RL AGNs, i.e. of blazars, significantly broadens the distribution. Second, the type of blazars included in the analysis changes the observed distribution of $\mathrm{X}$-ray spectral indices: if the sample selection favours objects with low values of $\alpha_{\mathrm{RX}}$ (like in a typical X-ray selected survey), the distribution will be more populated towards steep X-ray indices. Samples that include more sources with large values of $\alpha_{\mathrm{RX}}$ (like in a typical radio survey) are likely to include more blazars with a flat X-ray spectral index.

Acknowledgements. This research has made use of the NASA/IPAC Extragalactic Database (NED) which is operated by the Jet Propulsion Laboratory, California Institute of Technology, under contract with the National Aeronautics and Space Administration. E.G., A.C., T.M., V.B., R.D.C. and P.S. acknowledge partial financial support by the Italian Space Agency (ASI grants: I/R/062/02 and I/R/071/02) and by the MIUR (Cofin-03-02-23). P.S. and A.C. acknowledge financial support by the Istituto Nazionale di Astrofisica (INAF). Based on observations collected at the Italian "Telescopio Nazionale Galileo" (TNG), at the German-Spanish Astronomical Center, Calar Alto (operated jointly by Max-Planck Institut für Astronomie and Instituto de Astrofisica de Andalucia, CSIC) and at the European Southern Observatory (ESO). The TNG telescope is operated in the island of La Palma by the Centro Galileo Galilei of the INAF in the Spanish Observatorio del Roque de los Muchachos of the Instituto de Astrofísica de Canarias. We would like to thank C. Paizis, M. J. M. Marchã, A. Wolter and L. Ballo for useful suggestions and comments. We finally thank the APM team for maintaining their facility.

\section{References}

Avny, Y., Soltan, H., Tananbaum, H., \& Zamorani, G. 1980, ApJ, 238, 800

Ballantyne, D. R., Ross, R. R., \& Fabian, A. C. 2002, MNRAS, 332, L45

Becker, R. H., White, R. L., \& Helfand, D. J. 1995, AJ, 450, 559

Beckmann, V., Wolter, A., Celotti, A., et al. 2002, A\&A, 383, 410

Brocksopp, C., Puchnarewicz, E. M., Mason, K. O., Córdova, F. A., \& Priedhorsky, W. C. 2004, MNRAS, 349, 687

Brunner, H., Lamer, G., Worrall, D. M., \& Staubert, R. 1994, A\&A, 287, 436

Caccianiga, A., Maccacaro, T., Wolter, A., Della Ceca, R., \& Gioia, I. M. 1999, ApJ, 513, 51

Caccianiga, A., Marchã, M. J. M., Antón, A., Mack, K.-H., \& Neeser, M. 2001, MNRAS, 329, 877

Caccianiga, A., Severgnini, P., Braito, et al. 2004, A\&A, 416, 901

Cagnoni, I., Elvis, M., Kim, D. W., et al. 2001, ApJ, 560, 86

Canizares, C. R., \& White, J. L. 1989, ApJ, 339, 27

Cavallotti, F., Wolter, A., Stocke, J. T., \& Rector, T. 2004, A\&A, 419, 459

Ciliegi, P., Vignali, C., Comastri, A., et al. 2003, MNRAS, 342, 575

Cirasuolo, M., Celotti, A., Magliocchetti, M., \& Danese, L. 2003, MNRAS, 346, 447

Condon, J. J., Cotton, W. D., Greisen, E. W., et al. 1998, AJ, 115, 1693

Della Ceca, R., Zamorani, G., Maccacaro, et al. 1994, ApJ, 430, 533

Della Ceca, R., Braito, V., Beckmann, V., et al. 2003, A\&A, 406, 555

Della Ceca, R., Maccacaro, T., Caccianiga, A., et al. 2004, A\&A, in press

Dickey, J., \& Lockman, F. J. 1990, ARA\&A, 28, 215

Donato, D., Ghisellini, G., Tagliaferri, G., \& Fossati, G. 2001, A\&A, 375,739

Douglas, J. N., Bash, F. N., Boyzan, F. A., Torrence, G. W., \& Wolfe, C. 1996, AJ, 111, 1945

Ebeling, H., Jones, L. R., Fairley, B. W., et al. 2001, ApJ, 548, 23

Eracleous, M., Sambruna, R., \& Mushotzky, R. F. 2000, ApJ, 537, 654

Fabbiano, G., Trinchieri, G., Elvis, M., Miller, L., \& Longair, M. 1984, ApJ, 277, 115

Fabbiano, G. 1989, ARA\&A, 27, 87

Ferrero, E., \& Brinkmann, W. 2003, A\&A, 402, 465

Fiore, F., Brusa, M., Cocchia, F., et al. 2003, A\&A, 409, 79 
Franceschini, A., Vercellone, S., \& Fabian, A. C. 1998, MNRAS, 297, 817

Gambill, J. K., Sambruna, R. M., Chartas, G., et al. 2003, A\&A, 401, 505

Giommi, P., Menna, M. T., \& Padovani, P. 1999, MNRAS, 310, 465

Gregory, P. C., Scott, W. K., Douglas, K., \& Condon, J. J. 1996, ApJS, 103,427

Kellermann, K. I., Sramek, R., Schmidt, M., Shaffer, D. B., \& Green, R. 1989, AJ, 98, 1195

Hasenkopf, C. A., Sambruna, R. M., \& Eracleous, M. 2002, ApJ, 575, 127

Ho, L. C. 2002, ApJ, 564, 120

Ho, L. C., \& Peng, C. Y. 2001, ApJ, 555, 650

Ho, L. C., Filippenko, A. V., \& Sargent, W. L. W. 1997, ApJS, 112, 315

Ivezic, Z., Menou, K., Knapp, G. R., et al. 2002, AJ, 124, 2364

Joy, M., LaRoque, S., Grego, L., et al. 2001, ApJ, 551, L1

Lamer, G., Brunner, H., \& Staubert, R. 1996, A\&A, 311, 384

Landt, H., Padovani, P., Perlman, E. S., et al. 2001, MNRAS, 323, 757

Landt, H., Padovani, P., \& Giommi, P. 2002, MNRAS, 336, 945

Laor, A. 2000, ApJ, 543, 111

Laurent-Muehleisen, S. A., Kollgaard, R. I., Ciardullo, R., et al. 1998, ApJS, 118, 127

Londish, D., Heidt, J., Boyle, B. J., Croom, S. M., \& Kedziora-Chudczer, L. 2004, MNRAS, 352, 903

Maccacaro, T., Gioia, I. M., Wolter, A., Zamorani, G., \& Stocke, J. T. 1988, ApJ, 326, 680

Maccacaro, T., Caccianiga, A., Della Ceca, A., Wolter, A., \& Gioia, I. M. 1998, AN, 319, 15

Marchã, M. J. M., Browne, I. W. A., Impey, C. D., \& Smith, P. S. 1996, MNRAS, 281, 425

Marchã, M. J. M., Caccianiga, A., Browne, I. W. A., \& Jackson, N. 2001, MNRAS, 326, 1455

Meier, D. L. 1999, ApJ, 522, 753
Morris, S. L., Stocke, J. T., Gioia, I. M., et al. 1991, ApJ, 380, 49

Nagao, T., Murayama, T., \& Taniguchi, Y. 2001, ApJ, 546, 744

Owen, F. N., \& Ledlow, M. J. 1997, ApJS, 108, 41

Padovani, P., \& Giommi, P. 1996, MNRAS, 279, 526

Padovani, P., Giommi, P., \& Fiore, F. 1997, MNRAS, 284, 569

Padovani, P., Costamante, L., Giommi, P., et al. 2001, MNRAS, 328, 931

Padovani, P., Costamante, L., Giommi, P., et al. 2004, MNRAS, 347, 1282

Page, K. L., Turner, M. J. L., Done, C., et al. 2004, MNRAS, 349, 57

Perlman, E. S., Padovani, P., Giommi, P., et al. 1998, AJ, 115, 1253

Reeves, J. N., \& Turner, M. J. L. 2000, MNRAS, 316, 234

Reeves, J. N., Turner, M. J. L., Ohashi, T., \& Kii, T. 1997, MNRAS, 292, 468

Rengelink, R. B., Tang, Y., De Bruyn, A. G., et al. 1997, A\&AS, 124, 259

Severgnini, P., Caccianiga, A., Braito, et al. 2003, A\&A, 406, 483

Sambruna, R. M., Eracleous, M., \& Mushotzky, R. 1999, ApJ, 526, 60

Schwartz, D. A. 2004, in Proc. JENAM-2003 Symp., "Radio Astronomy at 70: from Karl Jansky to microjansky", Budapest, Hungary, 27-30 August 2003, ed. L. Gurvits, S. Frey, \& S. Rawlings (Les Ulis: EDP Sciences)

Shastri, P., Wilkes, B. J., Elvis, M., \& McDowell, J. 1993, ApJ, 410, 29

Stocke, J. T., Morris, S. L., Gioia, I., et al. 1990, ApJ, 348, 141

Urry, C. M., \& Padovani, P. 1995, PASP, 107, 803

Urry, C. M., Sambruna, R. M., Worrall, D. M., et al. 1996, ApJ, 463, 424

Visvanathan, N., \& Wills, B. J. 1998, AJ, 116, 2119

White, R. L., Becker, R. H., Gregg, M. D., et al. 2000, ApJS, 126, 133

Wilkes, B. J., \& Elvis, M. 1987, ApJ, 323, 243

Wolter, A., Comastri, A., Ghisellini, G., et al. 1998, A\&A, 335, 899

Wozniak, P. R., Zdziarski, A. A., Smith, D., Madejski, G. M., \& Johnson, W. N. 1998, MNRAS, 299, 449 


\section{Online Material}


E. Galbiati et al.: XMM-Newton spectroscopy of RL AGNs, Online Material $p 2$

\section{Appendix A: The X-ray spectra}
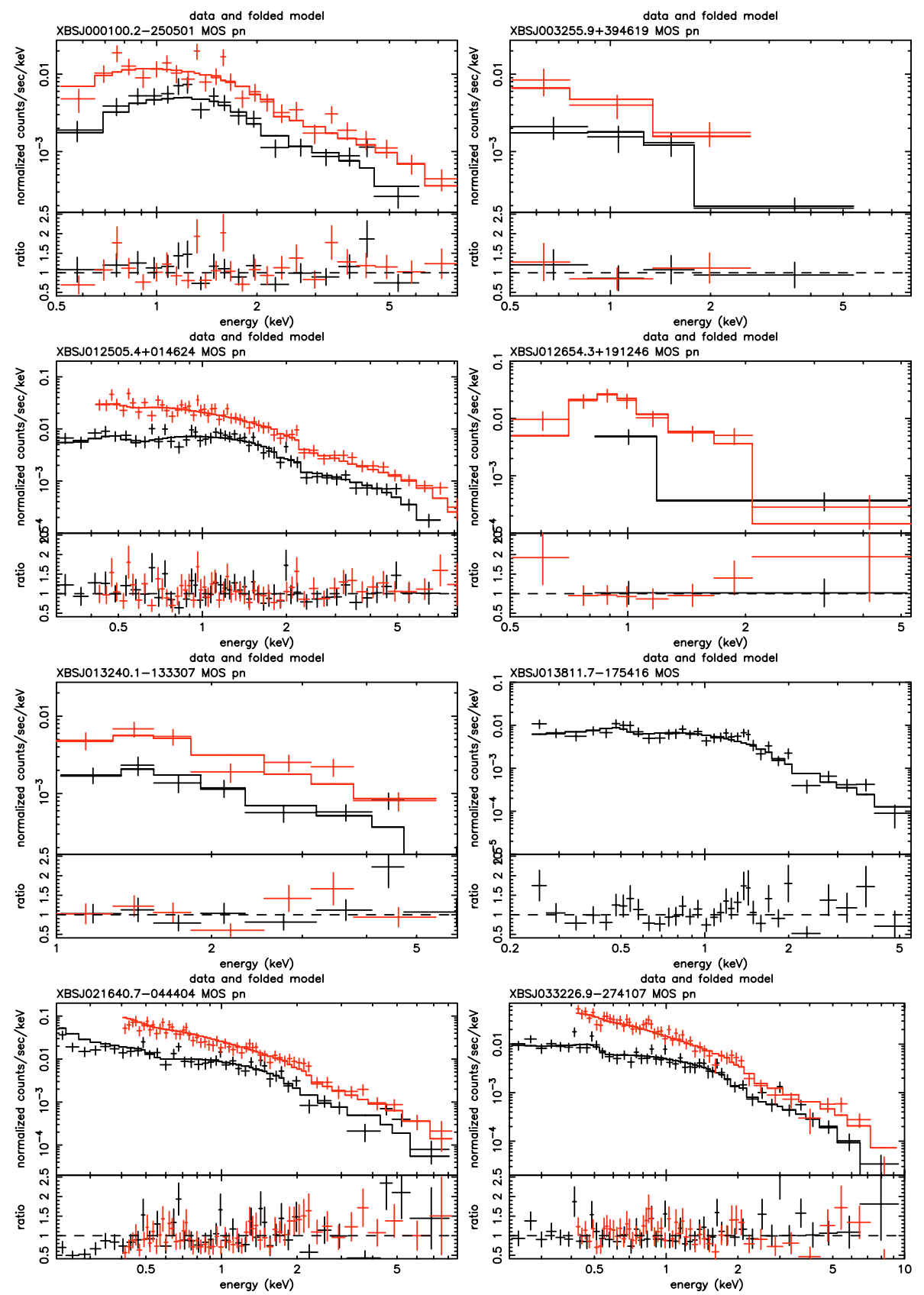

Fig. A.1. The best-fit X-ray spectra of the $25 \mathrm{RL}$ sources in the sample. Data (points) and folded model (continuous lines) are reported. 
E. Galbiati et al.: XMM-Newton spectroscopy of RL AGNs, Online Material $p 3$
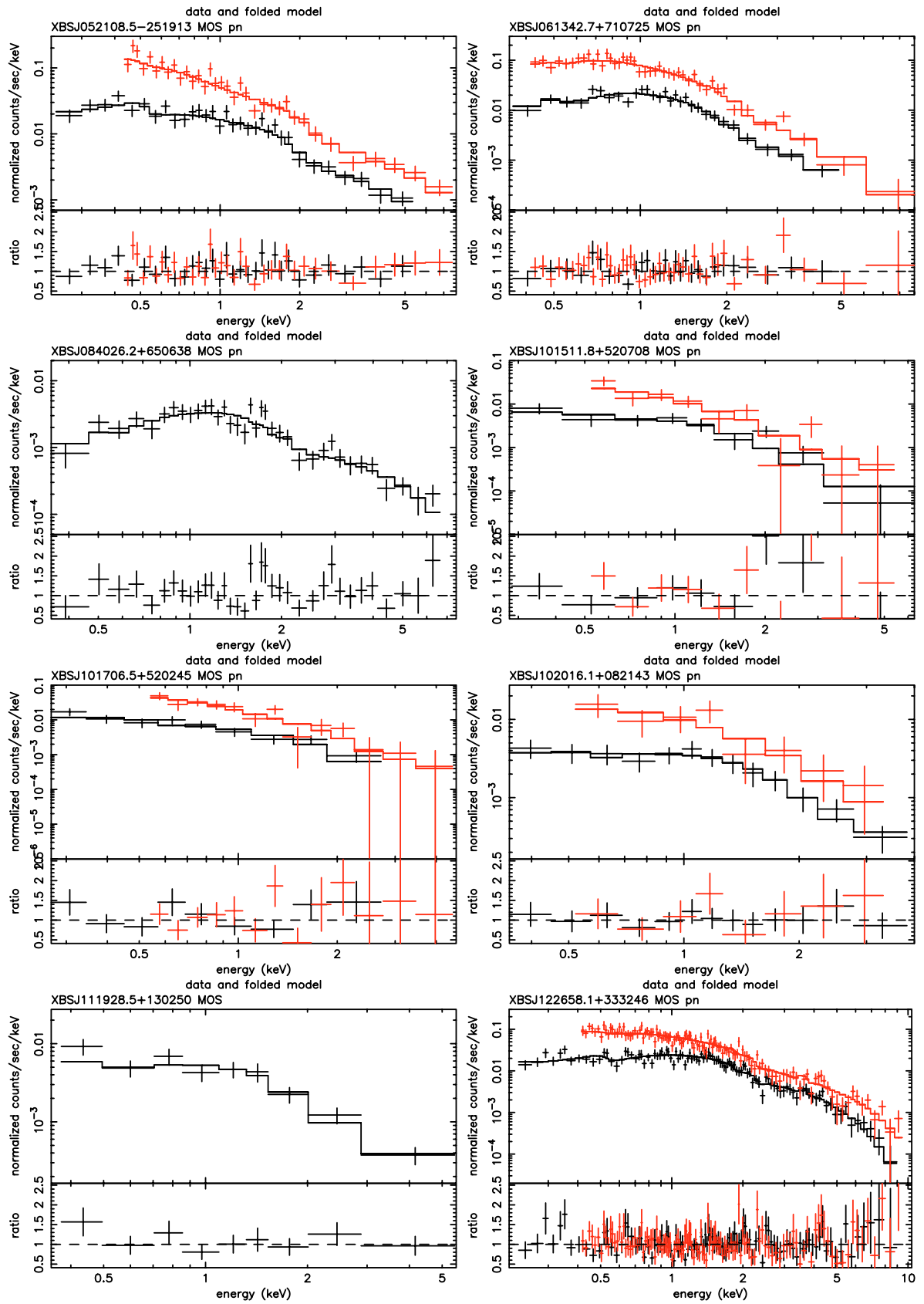

Fig. A.1. continued. 
E. Galbiati et al.: XMM-Newton spectroscopy of RL AGNs, Online Material p 4
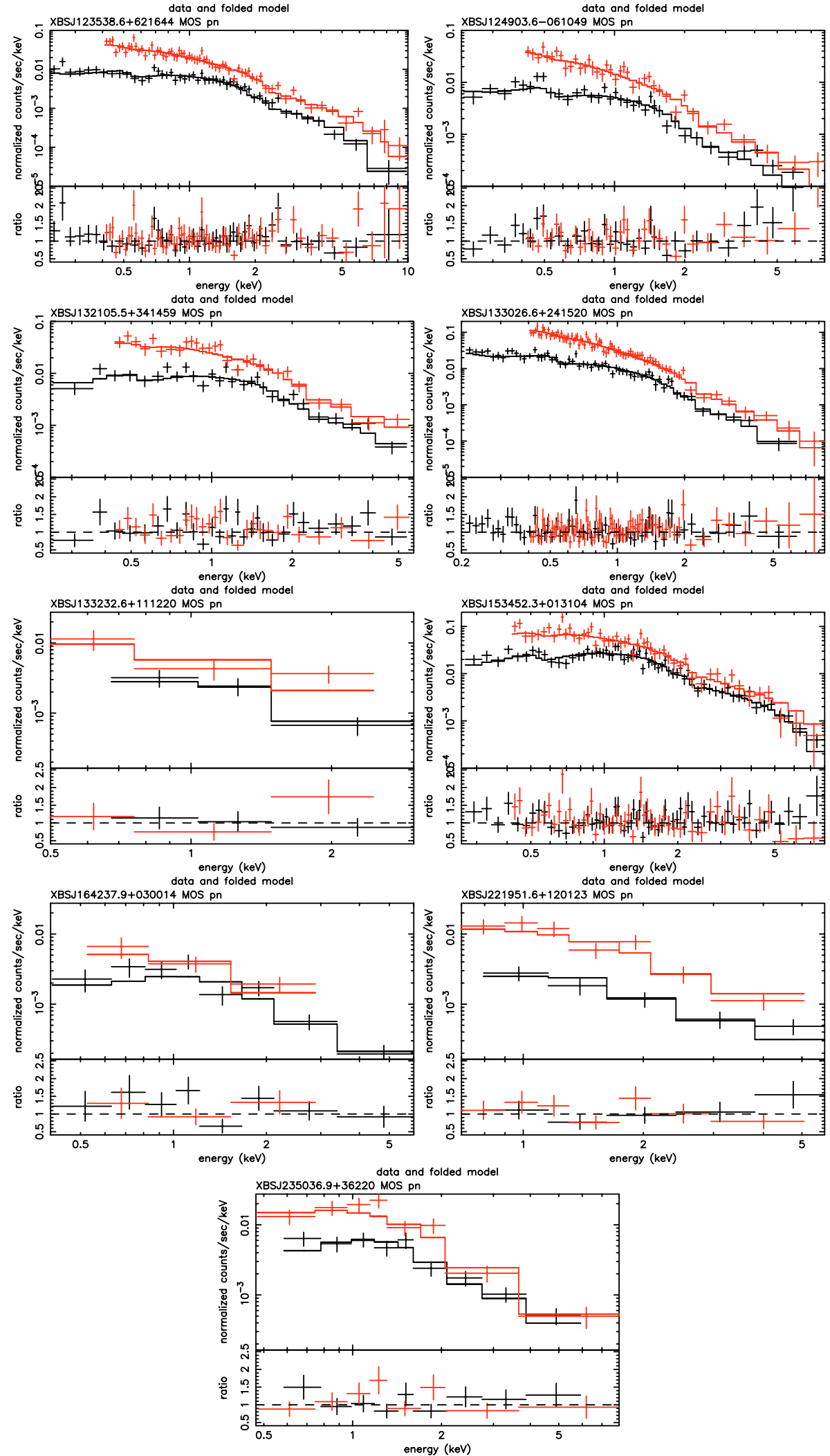

Fig. A.1. continued. 